\title{
Baseline PET/CT imaging parameters for prediction of treatment outcome in Hodgkin and diffuse large B cell lymphoma: a systematic review
}

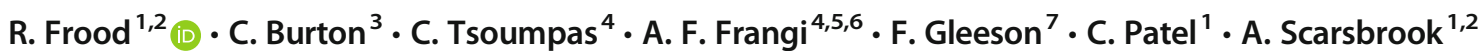

Received: 30 September 2020 / Accepted: 1 February 2021 / Published online: 18 February 2021

(C) The Author(s) 2021

\begin{abstract}
Purpose To systematically review the literature evaluating clinical utility of imaging metrics derived from baseline fluorine-18 fluorodeoxyglucose positron emission tomography/computed tomography (PET/CT) for prediction of progression-free (PFS) and overall survival (OS) in patients with classical Hodgkin lymphoma (HL) and diffuse large B cell lymphoma (DLBCL).

Methods A search of MEDLINE/PubMed, Web of Science, Cochrane, Scopus and clinicaltrials.gov databases was undertaken for articles evaluating PET/CT imaging metrics as outcome predictors in HL and DLBCL. PRISMA guidelines were followed. Risk of bias was assessed using the Quality in Prognosis Studies (QUIPS) tool.

Results Forty-one articles were included (31 DLBCL, $10 \mathrm{HL}$ ). Significant predictive ability was reported in 5/20 DLBCL studies assessing SUVmax (PFS: HR 0.13-7.35, OS: HR 0.83-11.23), 17/19 assessing metabolic tumour volume (MTV) (PFS: HR 2.0911.20, OS: HR 2.40-10.32) and 10/13 assessing total lesion glycolysis (TLG) (PFS: HR 1.078-11.21, OS: HR 2.40-4.82). Significant predictive ability was reported in 1/4 HL studies assessing SUVmax (HR not reported), 6/8 assessing MTV (PFS: HR 1.2-10.71, OS: HR 1.00-13.20) and 2/3 assessing TLG (HR not reported). There are 7/41 studies assessing the use of radiomics (4 DLBCL, $2 \mathrm{HL);}$ $5 / 41$ studies had internal validation and 2/41 included external validation. All studies had overall moderate or high risk of bias.

Conclusion Most studies are retrospective, underpowered, heterogenous in their methodology and lack external validation of described models. Further work in protocol harmonisation, automated segmentation techniques and optimum performance cutoff is required to develop robust methodologies amenable for clinical utility.
\end{abstract}

Keywords Diffuse large B-cell lymphoma $\cdot$ Hodgkin lymphoma $\cdot$ PET-CT $\cdot$ Outcome prediction $\cdot$ Radiomics

This article is part of the Topical Collection on Oncology - General.

R. Frood

russellfrood@nhs.net

1 Department of Nuclear Medicine, Leeds Teaching Hospitals NHS Trust, Leeds, UK

2 Leeds Institute of Health Research, University of Leeds, Leeds, UK

3 Department of Haematology, Leeds Teaching Hospitals NHS Trust, Leeds, UK

4 Leeds Institute of Cardiovascular and Metabolic Medicine, University of Leeds, Leeds, UK

5 Centre for Computational Imaging and Simulation Technologies in Biomedicine (CISTIB), School of Computing and School of Medicine, University of Leeds, Leeds, UK

6 Medical Imaging Research Center (MIRC), University Hospital Gasthuisberg, KU Leuven, Leuven, Belgium

7 Department of Radiology, Oxford University Hospitals NHS Foundation Trust, Oxford, UK

\section{Background}

Lymphoma is a haematopoietic malignancy, which can be broadly categorised into Hodgkin and non-Hodgkin disease. Hodgkin lymphoma (HL) accounts for approximately $10 \%$ of all newly diagnosed cases, and its hallmark is the presence of Hodgkin and Reed-Sternberg (HRS) cells [1]. HL can be further sub-divided based on morphology and immunohistochemistry into classical Hodgkin lymphoma (cHL), which has four further sub-categories, or nodular lymphocytepredominant Hodgkin lymphoma (NLPHL) [1]. The majority (90\%) of disease is due to cHL. HL is associated with a good prognosis having an overall 5-year survival of $86.6 \%$ [2]. Non-Hodgkin lymphoma (NHL) is the most prevalent form of lymphoma with over 50 sub-types, the most common being diffuse large B cell lymphoma (DLBCL) [3]. The overall 5year survival rate is $72 \%$ for NHL but this varies by stage and subtype [2]. DLBCL has a 5-year survival of approximately 
60-80\%, which has improved since the use of anthracyclinecontaining chemotherapy and rituximab (R-CHOP) [2, 4].

There are several pretreatment clinical prognostic tools developed to stratify both DLBCL and HL. In 1993, Shipp et al. introduced the international prognostic index (IPI) for predicting overall survival in DLBCL patients based on a retrospective study of 2031 patients treated with CHOP. The IPI has been further refined with an age-adjusted version (aaIPI), a revised version developed following the use of RCHOP (R-IPI), and a version based on the National Comprehensive Cancer Network database (NCCN-IPI). HL disease can be split into early (stage I and II) or advanced (stage III or stage IV) with early being split into favourable or unfavourable depending on one of the many scoring systems including, but not limited to, the German Hodgkin Study Group (GHSG), European Organisation of Research and Treatment of Cancer (EORTC), Groupe d'Etudes des Lymphomes de l'Adulte (GELA), National Cancer Institute (NCI) or National Comprehensive Cancer Network 2010 (NCCN 2010) scores. However, given the variation in the prognostic groups derived from the different scoring systems, further information obtained from imaging may improve prognostication.

2-deoxy-2-[Fluorine-18]fluoro-D-glucose (FDG) positron emission tomography/computed tomography (PET/CT) is widely used for staging and response assessment in $\mathrm{HL}$ and NHL [5]. Response assessment PET/CT studies are performed at various time points, including during and after treatment [5]. The parameter most commonly used in assessment is the standardised uptake value (SUV) at sites of disease, which is compared to physiological activity in reference areas such as the mediastinal blood pool and liver and is reported using an ordinal (qualitative) scale (Deauville Score (DS)).

A variety of imaging-derived quantitative parameters have been reported in the literature with potential utility for predicting prognosis or treatment outcome. These metrics range from those based on tumour volume to metabolic features, including shape and texture. At present, none have been translated into routine clinical practice. The purpose of this study was to perform a systematic review of the literature reporting the use of quantitative imaging parameters derived from pretreatment FDG PET/CT for prediction of treatment outcome for HL and DLBCL. Due to the varied nature of NHL, DLBCL was chosen as it is the most common subtype of NHL.

\section{Methods}

\section{Search strategy and selection criteria}

A search of MEDLINE/PubMed, Web of Science, Cochrane, Scopus and clinicaltrials.gov databases was performed for articles on PET/CT imaging parameters in lymphoma treatment assessment. The search strategy included three primary operator criteria linked with the "AND" function. The first criteria consisted of "lymphoma", the second of "PET" or "positron emission tomography", and the third of "outcome", "prognosis", "prediction", "parameter", "radiomics", "machine learning", "deep learning" or "artificial intelligence". Case studies, articles not published in English, phantom studies, studies not assessing treatment outcomes using baseline imaging in HL or DLCBL, studies assessing primary anatomical presentations of lymphoma or HIV-related lymphoma, mixed pathology studies and studies assessing novel treatments were excluded. After duplications were excluded, studies were screened for eligibility based on the title, abstract and subsequently on full text. The references of the articles included in the systematic review were manually reviewed to identify further publications which met the inclusion criteria. The results were stored in bibliographic management software. Preferred Reporting Items for Systematic Reviews and Meta-Analysis (PRISMA) criteria were adhered to [6].

\section{Quality assessment}

The Quality in Prognosis Studies (QUIPS) tool was used to evaluate validity and bias which considers six areas: inclusion, attrition, prognostic factor measurement, confounders, outcome measurement, and analysis and reporting [7]. Prompting questions and modifications applied to the QUIPS tool are detailed in Supplemental Table 1. Two authors (RF and AS) independently reviewed all studies which met inclusion criteria and scored each of the six domains as high, moderate or low risk of bias. Any discrepancies were agreed in consensus. Overall risk of bias for each paper was further categorised based on the following criteria: if all domains were classified as low risk, or there was up to one moderate risk, the paper was classified as low risk of bias. If one or more domains were classified as high risk, the paper was classified as high risk of bias. All papers in between were classified as having moderate risk of bias [8].

\section{Results}

Results are current to July 2020. The database search strings yielded 2717 results after duplicates were excluded. Following screening and assessment of eligibility, 41 articles meeting the study inclusion criteria were included. Figure 1 details the study selection.

\section{Quality assessment}

No studies showed low risk of bias in all six domains (Supplemental Table 2). Only two studies demonstrated a 
Fig. 1 PRISMA flow diagram illustrating the methodology for study selection for the systematic review of lymphoma imaging parameters. $B M I$ bone marrow involvement, Relapse indicates studies investigating previously treated cases

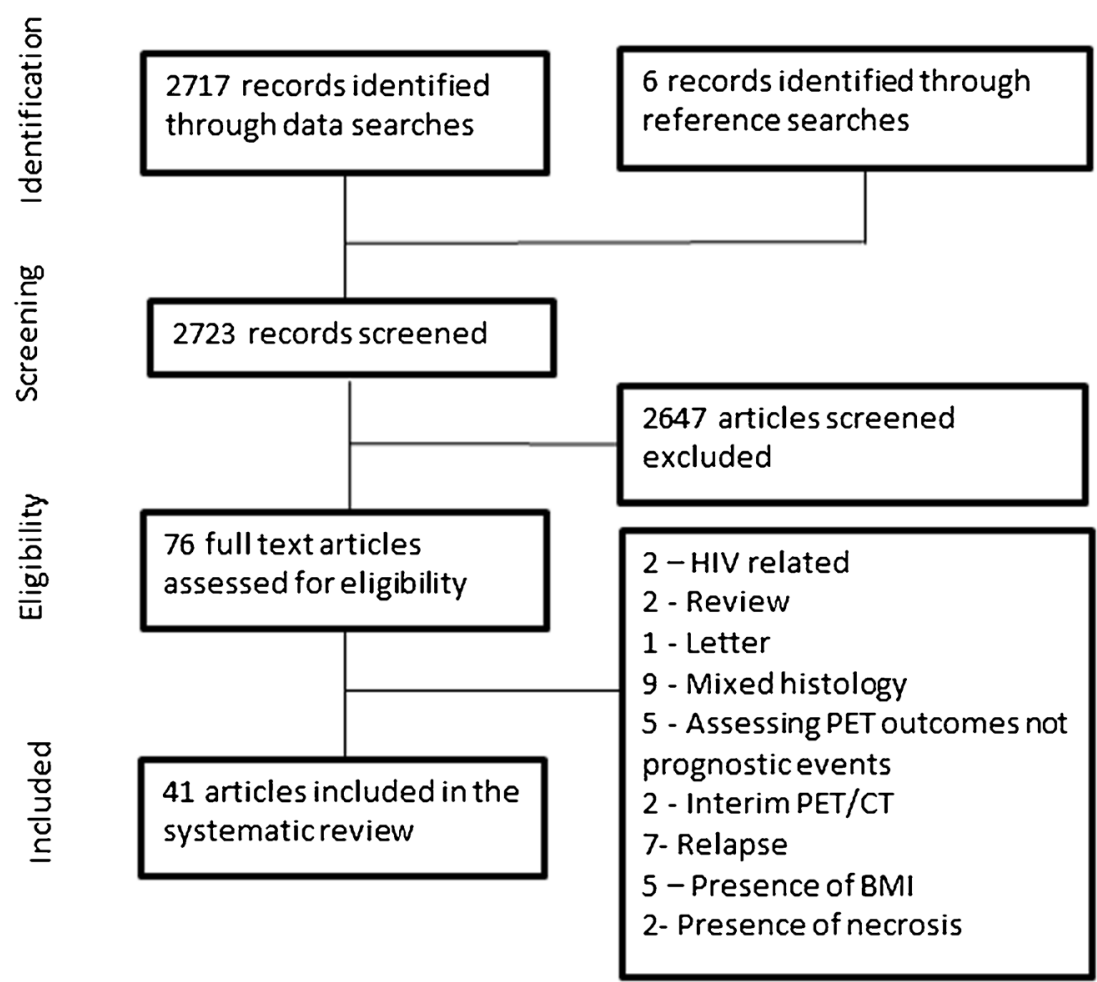

low risk for participation; no studies had a low risk in attrition, prognostic measurement, outcome measurement or confounding factors; 33 studies had low risk for analysis and reporting. All studies were assessed as having either moderate (24/41, $59 \%)$ or high $(17 / 41,41 \%)$ overall risk of bias. Of the high risk studies, 6 had high risk scores of bias in participation, 5 in attrition, 8 in prognostic measurement, 8 in outcome measurement, 10 in confounding factors and 7 in analysis and reporting categories.

All studies were retrospective, with 28/41 single centre. Six reports were based on retrospective analysis of trial data from prospective studies. Four studies stated that they were compliant with the European Association of Nuclear Medicine (EANM) guidelines with their scanning protocol; 10/41 did not take into consideration important co-founders such as different treatment regimes, stage, prognostic scores or histology. Only six studies defined the method for calculation of SUV, and 7 studies used a validation cohort to test the predictive models (Table 1). Of the radiomic studies, one study referenced the image biomarker standardisation initiative (IBSI) within the discussion but none of the papers explicitly stated that they had complied with IBSI guidelines.

As there were no studies deemed to be of low risk for overall bias, a decision was made to include the high risk studies in the systematic review, as removal of these would introduce its own inherent bias.

\section{Metabolic parameters}

SUV is the commonest metric extracted from PET studies. This represents a ratio of radioactivity at a given image location compared to injected whole-body radioactivity [50]. There are several iterations of SUV, including the maximum or mean SUV within a contoured area (SUVmax and SUVmean), or SUVpeak which is the average SUV of a region of interest centred on the highest uptake region within the contoured area. SUV supports other metabolic parameters such as metabolic tumour volume (MTV), which is the volume of disease contoured at a specified SUV threshold, and total lesion glycolysis (TLG), which is the MTV multiplied by SUVmean. Published evidence regarding metabolic parameters used in the pretreatment assessment of lymphoma is summarised below.

\section{SUV metrics for prediction of outcome}

\section{a) $\mathrm{DLBCL}$}

The majority of studies assessing the use of baseline SUVmax in DLBCL report no significant ability to predict progression-free survival (PFS) or overall survival (OS) (Table 2). Forest plots illustrating hazard ratios (HR) for PFS and OS are demonstrated in Figs. 2 and 3. From the results included in the forest, the overall 
Table 1 Overview of study design and risk of bias for each of the studies included in the systematic review

\begin{tabular}{|c|c|c|c|c|c|c|c|c|c|}
\hline Study & Prospective & $\begin{array}{l}\text { Multi- } \\
\text { centre }\end{array}$ & $\begin{array}{l}\text { PET scanners } \\
\text { used }\end{array}$ & $\begin{array}{l}\text { EANM } \\
\text { guidelines } \\
\text { stated }\end{array}$ & $\begin{array}{l}\text { SUV } \\
\text { defined }\end{array}$ & $\begin{array}{l}\text { Definition of prognostic factor } \\
\text { provide }\end{array}$ & $\begin{array}{l}\text { Follow up } \\
\text { period }\end{array}$ & $\begin{array}{l}\text { Separate } \\
\text { validation } \\
\text { cohort }\end{array}$ & $\begin{array}{l}\text { Overall } \\
\text { risk of } \\
\text { bias }\end{array}$ \\
\hline Adams [9] & $\mathrm{N}$ & $\mathrm{N}$ & $\begin{array}{l}\text { Siemens } \\
\text { Biograph } 40 \\
\text { TruePoint }\end{array}$ & $\mathrm{N}$ & $\mathrm{N}$ & $\begin{array}{l}\text { PFS - } \\
\text { relapse/progression/death } \\
\text { attributable to PFS } \\
\text { OS - Death from any cause }\end{array}$ & $\begin{array}{l}\text { Median: } \\
994 \text { days }\end{array}$ & $\mathrm{N}$ & Moderate \\
\hline Aide [10] & $\mathrm{N}$ & $\mathrm{N}$ & $\begin{array}{l}\text { Siemens Biograph } \\
\text { TrueV }\end{array}$ & $\mathrm{N}$ & $\mathrm{N}$ & $\begin{array}{l}\text { EFS - } \\
\text { relapse/progression/- } \\
\text { unplanned treatment/death } \\
\text { attributable to PFS }\end{array}$ & 2-year EFS & $\mathrm{Y}$ & High \\
\hline Aide [11] & $\mathrm{N}$ & $\mathrm{N}$ & $\begin{array}{l}\text { Siemens Biograph } \\
\text { TrueV }\end{array}$ & Y & $\mathrm{N}$ & $\begin{array}{l}\text { PFS - relapse/progression } \\
\text { OS - death from lymphoma or } \\
\text { treatment }\end{array}$ & $\begin{array}{l}\text { Median: } \\
25.7 \text { mont- } \\
\text { hs }\end{array}$ & $\mathrm{N}$ & Moderate \\
\hline Akhtari [12] & $\mathrm{N}$ & $\mathrm{N}$ & $\begin{array}{l}\text { GE Discovery ST } \\
\text { GE Discovery } \\
\text { RX GE } \\
\text { Discovery STE }\end{array}$ & $\mathrm{N}$ & $\mathrm{Y}(\mathrm{bw})$ & $\begin{array}{l}\text { FFP - relapse or refractory } \\
\text { disease } \\
\text { OS - death from any cause }\end{array}$ & $\begin{array}{l}\text { Median: } \\
4.96 \text { years }\end{array}$ & $\mathrm{N}$ & Moderate \\
\hline Albano [13] & $\mathrm{N}$ & $\mathrm{Y}$ & $\begin{array}{l}\text { GE Discovery ST } \\
\text { GE Discovery } \\
690\end{array}$ & Y & $\mathrm{N}$ & $\begin{array}{l}\text { PFS - } \\
\text { progression/relapse/death } \\
\text { OS - death from any cause }\end{array}$ & $\begin{array}{l}\text { Median: } \\
\quad 40 \text { months }\end{array}$ & $\mathrm{N}$ & Moderate \\
\hline $\begin{array}{l}\text { Angelopulou } \\
{[14]}\end{array}$ & $\mathrm{N}$ & $\mathrm{N}$ & $\begin{array}{l}\text { Multiple not } \\
\text { defined }\end{array}$ & $\mathrm{N}$ & $\mathrm{N}$ & $\begin{array}{l}\text { FFP - relapse or refractory } \\
\text { disease } \\
\text { OS - death from any cause }\end{array}$ & $\begin{array}{l}\text { Median: } \\
56 \text { months }\end{array}$ & $\mathrm{N}$ & High \\
\hline $\begin{array}{l}\text { Capobianco } \\
{[15]}\end{array}$ & $\mathrm{N}$ & $\mathrm{Y}$ & Multiple $^{1}$ & $\mathrm{~N}$ & $\mathrm{~N}$ & Not defined & $\begin{array}{l}\text { Median: } \\
5 \text { years }\end{array}$ & $\mathrm{Y}$ & High \\
\hline Ceriani [16] & $\mathrm{N}$ & $\mathrm{Y}$ & $\begin{array}{l}\text { Multiple not } \\
\text { defined }\end{array}$ & $\mathrm{N}$ & $\mathrm{N}$ & Not defined & $\begin{array}{l}\text { Median: } \\
64 \text { months, } \\
34 \text { months }\end{array}$ & $\mathrm{Y}$ & High \\
\hline Chang [17] & $\mathrm{N}$ & $\mathrm{N}$ & GE Discovery ST & $\mathrm{N}$ & $\mathrm{N}$ & $\begin{array}{l}\text { PFS - } \\
\text { progression/relapse/death } \\
\text { OS - death from any cause }\end{array}$ & $\begin{array}{l}\text { Median: } \\
28.7 \text { mont- } \\
\text { hs }\end{array}$ & $\mathrm{N}$ & Moderate \\
\hline Chang [18] & $\mathrm{N}$ & $\mathrm{N}$ & GE Discovery ST & $\mathrm{N}$ & $\mathrm{N}$ & $\begin{array}{l}\text { PFS - } \\
\text { progression/relapse/death } \\
\text { OS - death from any cause }\end{array}$ & $\begin{array}{l}\text { median } \\
36 \text { months }\end{array}$ & $\mathrm{N}$ & Moderate \\
\hline Chihara [19] & $\mathrm{N}$ & $\mathrm{N}$ & GE Discovery LS & $\mathrm{N}$ & $\mathrm{Y}(\mathrm{bw})$ & $\begin{array}{l}\text { PFS - } \\
\text { progression/relapse/death } \\
\text { from any cause OS - death } \\
\text { from any cause }\end{array}$ & $\begin{array}{l}\text { Median: } \\
34.4 \text { mont- } \\
\text { hs }\end{array}$ & $\mathrm{N}$ & Moderate \\
\hline $\begin{array}{l}\text { Cottereau } \\
{[20]}\end{array}$ & $\mathrm{N}$ & $\mathrm{Y}$ & $\begin{array}{l}\text { Multiple not } \\
\text { defined }\end{array}$ & $\mathrm{N}$ & $\mathrm{N}$ & Not defined & $\begin{array}{l}\text { Median: } \\
\quad 44 \text { months }\end{array}$ & $\mathrm{N}$ & High \\
\hline $\begin{array}{l}\text { Cottereau } \\
{[21]}\end{array}$ & $\mathrm{N}$ & $\mathrm{Y}$ & $\begin{array}{l}\text { Multiple not } \\
\text { defined }\end{array}$ & $\mathrm{N}$ & $\mathrm{N}$ & $\begin{array}{l}\text { PFS - progression/death from } \\
\text { any cause } \\
\text { OS - death from any cause }\end{array}$ & $\begin{array}{l}\text { Median: } \\
\quad 55 \text { months }\end{array}$ & $\mathrm{Y}$ & Moderate \\
\hline $\begin{array}{l}\text { Cottereau } \\
{[22]}\end{array}$ & $\mathrm{N}$ & $\mathrm{N}$ & $\begin{array}{l}\text { Siemens } \\
\text { Biograph } 16\end{array}$ & $\mathrm{~N}$ & $\mathrm{~N}$ & $\begin{array}{l}\text { OS and PFS were defined } \\
\text { according to the revised NCI } \\
\text { criteria }\end{array}$ & $\begin{array}{l}\text { Median: } \\
64 \text { months }\end{array}$ & $\mathrm{N}$ & Moderate \\
\hline Decazes [23] & $\mathrm{N}$ & $\mathrm{N}$ & $\begin{array}{l}\text { Siemens Biograph } \\
\text { Sensation } 16 \\
\text { HiRes }\end{array}$ & $\mathrm{N}$ & $\mathrm{N}$ & $\begin{array}{l}\text { Both OS and PFS were defined } \\
\text { according to the revised NCI } \\
\text { criteria }\end{array}$ & $\begin{array}{l}\text { Median: } \\
44 \text { months }\end{array}$ & $\mathrm{N}$ & Moderate \\
\hline Esfahani [24] & $\mathrm{N}$ & $\mathrm{N}$ & Siemens Biograph & $\mathrm{N}$ & $\mathrm{N}$ & PFS - recurrence & $\begin{array}{l}\text { Mean: } \\
51 \text { months }\end{array}$ & $\mathrm{N}$ & High \\
\hline $\begin{array}{l}\text { Gallicchio } \\
{[25]}\end{array}$ & $\mathrm{N}$ & $\mathrm{N}$ & $\begin{array}{l}\text { GE Discovery } \\
\text { VCT GE } \\
\text { Discovery LS } \\
\text { VCT }\end{array}$ & $\mathrm{N}$ & $\mathrm{N}$ & $\begin{array}{l}\text { Progression/disease-related } \\
\text { death }\end{array}$ & $\begin{array}{l}\text { Median: } \\
18 \text { months }\end{array}$ & $\mathrm{N}$ & High \\
\hline Huang [26] & $\mathrm{N}$ & $\mathrm{N}$ & GE Discovery LS & $\mathrm{N}$ & $\mathrm{Y}(\mathrm{bw})$ & $\begin{array}{l}\text { PFS - } \\
\text { progression/relapse/death } \\
\text { OS - death from any cause }\end{array}$ & $\begin{array}{l}\text { Median: } \\
30 \text { months }\end{array}$ & $\mathrm{N}$ & Moderate \\
\hline Ilyas [27] & $\mathrm{N}$ & $\mathrm{N}$ & $\begin{array}{l}\text { GE Discovery ST } \\
\text { GE Discovery } \\
\text { VCT }\end{array}$ & $\mathrm{N}$ & $\mathrm{N}$ & $\begin{array}{l}\text { PFS - progression/death from } \\
\text { any cause } \\
\text { OS - death from any cause }\end{array}$ & $\begin{array}{l}\text { Median: } \\
3.8 \text { years }\end{array}$ & $\mathrm{N}$ & High \\
\hline Jegadesh [28] & $\mathrm{N}$ & $\mathrm{N}$ & Not defined & $\mathrm{N}$ & $\mathrm{N}$ & Not defined & & $\mathrm{N}$ & Moderate \\
\hline
\end{tabular}


Table 1 (continued)

\begin{tabular}{|c|c|c|c|c|c|c|c|c|c|}
\hline Study & Prospective & $\begin{array}{l}\text { Multi- } \\
\text { centre }\end{array}$ & $\begin{array}{l}\text { PET scanners } \\
\text { used }\end{array}$ & $\begin{array}{l}\text { EANM } \\
\text { guidelines } \\
\text { stated }\end{array}$ & $\begin{array}{l}\text { SUV } \\
\text { defined }\end{array}$ & $\begin{array}{l}\text { Definition of prognostic factor } \\
\text { provide }\end{array}$ & $\begin{array}{l}\text { Follow up } \\
\text { period }\end{array}$ & $\begin{array}{l}\text { Separate } \\
\text { validation } \\
\text { cohort }\end{array}$ & $\begin{array}{l}\text { Overall } \\
\text { risk of } \\
\text { bias }\end{array}$ \\
\hline & & & & & & & $\begin{array}{l}\text { Median: } \\
43.9 \text { mont- } \\
\text { hs }\end{array}$ & & \\
\hline Kanoun [29] & $\mathrm{N}$ & $\mathrm{N}$ & $\begin{array}{l}\text { Philips Gemini } \\
\text { GXL } \\
\text { Philips Gemini } \\
\text { TOF }\end{array}$ & $\mathrm{N}$ & $\mathrm{N}$ & $\begin{array}{l}\text { PFS - } \\
\text { progression/relapse/death } \\
\text { from any cause }\end{array}$ & $\begin{array}{l}\text { Median: } \\
50 \text { months }\end{array}$ & $\mathrm{N}$ & High \\
\hline $\operatorname{Kim}[30]$ & $\mathrm{N}$ & $\mathrm{N}$ & $\begin{array}{l}\text { Siemens } \\
\quad \text { Biograph } 6\end{array}$ & $\mathrm{~N}$ & $\mathrm{~N}$ & $\begin{array}{l}\text { EFS - } \\
\text { relapse/progression/stopping } \\
\text { of treatment/death from any } \\
\text { cause }\end{array}$ & $\begin{array}{l}\text { Median: } \\
27.8 \text { mont- } \\
\text { hs }\end{array}$ & $\mathrm{N}$ & Moderate \\
\hline $\operatorname{Kim}[31]$ & $\mathrm{N}$ & $\mathrm{N}$ & $\begin{array}{l}\text { Philips Gemini } \\
\text { Siemens Biograph } \\
\quad 40\end{array}$ & $\mathrm{~N}$ & $\mathrm{~N}$ & $\begin{array}{l}\text { OS - death from any cause } \\
\text { PFS - } \\
\text { progression/relapse/death } \\
\text { OS - death (? any cause) }\end{array}$ & $\begin{array}{l}\text { Median: } \\
25.8 \text { mont- } \\
\text { hs }\end{array}$ & $\mathrm{N}$ & Moderate \\
\hline Kwon [32] & $\mathrm{N}$ & $\mathrm{Y}$ & GE Discovery ST & $\mathrm{N}$ & $\mathrm{Y}(\mathrm{bw})$ & $\begin{array}{l}\text { PFS - } \\
\text { progression/relapse/death } \\
\text { from any cause }\end{array}$ & $\begin{array}{l}\text { Median: } \\
30.8 \text { mont- } \\
\text { hs }\end{array}$ & $\mathrm{N}$ & High \\
\hline Lanic [33] & $\mathrm{N}$ & $\mathrm{Y}$ & $\begin{array}{l}\text { Siemens Biograph } \\
\text { LSO Sensation } \\
16\end{array}$ & $\mathrm{~N}$ & $\mathrm{~N}$ & $\begin{array}{l}\text { OS - death from any cause } \\
\text { PFS - } \\
\text { progression/relapse/death } \\
\text { from any cause }\end{array}$ & $\begin{array}{l}\text { Median: } \\
28 \text { months }\end{array}$ & $\mathrm{N}$ & High \\
\hline Lue [34] & $\mathrm{N}$ & $\mathrm{N}$ & GE Discovery ST & $\mathrm{N}$ & $\mathrm{N}$ & $\begin{array}{l}\text { OS - death from any cause } \\
\text { PFS - } \\
\text { progression/relapse/death } \\
\text { from any cause }\end{array}$ & $\begin{array}{l}\text { Median: } \\
48 \text { months }\end{array}$ & $\mathrm{N}$ & Moderate \\
\hline Mettler [35] & $\mathrm{N}$ & Y & $\begin{array}{l}\text { Multiple not } \\
\text { defined }\end{array}$ & $\mathrm{N}$ & $\mathrm{N}$ & $\begin{array}{l}\text { OS - death from any cause } \\
\text { PFS - } \\
\text { progression/relapse/death } \\
\text { from any cause }\end{array}$ & Not defined & $\mathrm{N}$ & High \\
\hline $\begin{array}{l}\text { Mikhaeel } \\
{[36]}\end{array}$ & $\mathrm{N}$ & $\mathrm{N}$ & $\begin{array}{l}\text { GE Discovery ST } \\
\text { GE Discovery } \\
\text { VCT }\end{array}$ & $\mathrm{N}$ & $\mathrm{N}$ & $\begin{array}{l}\text { OS - death from any cause } \\
\text { PFS - progression/death from } \\
\text { any cause } \\
\text { OS - death }\end{array}$ & $\begin{array}{l}\text { Median: } \\
\quad 3.8 \text { years }\end{array}$ & $\mathrm{N}$ & Moderate \\
\hline Milgrom [37] & $\mathrm{N}$ & $\mathrm{N}$ & $\begin{array}{l}\text { GE Discovery ST } \\
\text { GE Discovery } \\
\text { RX GE } \\
\text { Discovery STE }\end{array}$ & $\mathrm{N}$ & $\mathrm{N}$ & Relapse or progression or death & Not defined & $\mathrm{Y}$ & High \\
\hline $\begin{array}{l}\text { Miyazaki } \\
\text { [38] }\end{array}$ & $\mathrm{N}$ & $\mathrm{N}$ & $\begin{array}{l}\text { GE Discovery } \\
\text { STE }\end{array}$ & $\mathrm{N}$ & $\mathrm{N}$ & $\begin{array}{l}\text { PFS - relapse/death from any } \\
\text { cause } \\
\text { OS - death }\end{array}$ & $\begin{array}{l}\text { Median: } \\
32.7 \text { mont- } \\
\text { hs }\end{array}$ & $\mathrm{N}$ & Moderate \\
\hline Park [39] & $\mathrm{N}$ & $\mathrm{N}$ & $\begin{array}{l}\text { GE Discovery LS, } \\
\text { GE Discovery } \\
\text { STE }\end{array}$ & $\mathrm{N}$ & $\mathrm{N}$ & $\begin{array}{l}\text { PFS - } \\
\text { progression/relapse/death } \\
\text { from any cause }\end{array}$ & $\begin{array}{l}\text { Median: } \\
21 \text { months }\end{array}$ & $\mathrm{N}$ & High \\
\hline Sasanelli [40] & $\mathrm{N}$ & $\mathrm{Y}$ & $\begin{array}{l}\text { Philips Gemini } \\
\text { GXL } \\
\text { Siemens }\end{array}$ & $\mathrm{N}$ & $\mathrm{Y}(\mathrm{bw})$ & $\begin{array}{l}\text { OS - death from any cause } \\
\text { PFS - relapse } \\
\text { OS - death from any cause }\end{array}$ & $\begin{array}{l}\text { Median: } \\
39 \text { months }\end{array}$ & $\mathrm{N}$ & Moderate \\
\hline Senjo [41] & $\mathrm{N}$ & $\mathrm{Y}$ & $\begin{array}{l}\text { Biograph } 2 \\
\text { GE Discovery ST } \\
\text { Philips Gemini } \\
\text { GXL } \\
\text { GE Discovery ST }\end{array}$ & $\mathrm{Y}$ & $\mathrm{Y}(\mathrm{bw})$ & $\begin{array}{l}\text { PFS - } \\
\text { progression/relapse/death } \\
\text { OS - death }\end{array}$ & $\begin{array}{l}\text { Median: } \\
33.1 \text { mont- } \\
\text { hs, } \\
32.8 \text { mont- } \\
\text { hs }\end{array}$ & $\mathrm{Y}$ & High \\
\hline Song [42] & $\mathrm{N}$ & $\mathrm{Y}$ & Siemens Biograph & $\mathrm{N}$ & $\mathrm{N}$ & $\begin{array}{l}\text { PFS progression OS - death } \\
\text { from any cause }\end{array}$ & $\begin{array}{l}\text { Median: } \\
40.8 \text { mont- } \\
\text { hs }\end{array}$ & $\mathrm{Y}$ & Moderate \\
\hline Song [43] & $\mathrm{N}$ & $\mathrm{Y}$ & Siemens Biograph & $\mathrm{N}$ & $\mathrm{N}$ & Not defined & & $\mathrm{N}$ & Moderate \\
\hline
\end{tabular}


Table 1 (continued)

\begin{tabular}{|c|c|c|c|c|c|c|c|c|c|}
\hline Study & Prospective & $\begin{array}{l}\text { Multi- } \\
\text { centre }\end{array}$ & $\begin{array}{l}\text { PET scanners } \\
\text { used }\end{array}$ & $\begin{array}{l}\text { EANM } \\
\text { guidelines } \\
\text { stated }\end{array}$ & $\begin{array}{l}\text { SUV } \\
\text { defined }\end{array}$ & $\begin{array}{l}\text { Definition of prognostic factor } \\
\text { provide }\end{array}$ & $\begin{array}{l}\text { Follow up } \\
\text { period }\end{array}$ & $\begin{array}{l}\text { Separate } \\
\text { validation } \\
\text { cohort }\end{array}$ & $\begin{array}{l}\text { Overall } \\
\text { risk of } \\
\text { bias }\end{array}$ \\
\hline & & & & & & & $\begin{array}{l}\text { Median: } \\
45.8 \text { mont- } \\
\text { hs }\end{array}$ & & \\
\hline Song [44] & $\mathrm{N}$ & $\mathrm{Y}$ & Siemens Biograph & $\mathrm{N}$ & $\mathrm{N}$ & $\begin{array}{l}\text { PFS progression, death related } \\
\text { to lymphoma }\end{array}$ & $\begin{array}{l}\text { Median: } \\
\quad 36 \text { months }\end{array}$ & $\mathrm{N}$ & Moderate \\
\hline $\begin{array}{l}\text { Toledano } \\
\text { [45] }\end{array}$ & $\mathrm{N}$ & $\mathrm{N}$ & $\begin{array}{l}\text { Siemens Biograph } \\
\text { Sensation } 16 \\
\text { HiRes }\end{array}$ & $\mathrm{N}$ & $\mathrm{N}$ & $\begin{array}{l}\text { OS - death from any cause } \\
\text { OS and PFS were defined } \\
\text { according to the revised NCI } \\
\text { criteria }\end{array}$ & $\begin{array}{l}\text { Median: } \\
40 \text { months }\end{array}$ & $\mathrm{N}$ & Moderate \\
\hline Tseng [46] & $\mathrm{N}$ & $\mathrm{N}$ & GE Discovery LS & $\mathrm{N}$ & $\mathrm{N}$ & Not defined & $\begin{array}{l}\text { Median: } \\
50 \text { months }\end{array}$ & $\mathrm{N}$ & High \\
\hline Xie [47] & $\mathrm{N}$ & $\mathrm{N}$ & $\begin{array}{l}\text { Siemens } \\
\text { Biograph } 64\end{array}$ & $\mathrm{Y}$ & $\mathrm{N}$ & $\begin{array}{l}\text { PFS - } \\
\text { progression/relapse/death } \\
\text { from any cause }\end{array}$ & $\begin{array}{l}\text { Median: } \\
17 \text { months }\end{array}$ & $\mathrm{N}$ & High \\
\hline Zhang [48] & $\mathrm{N}$ & $\mathrm{N}$ & $\begin{array}{l}\text { Siemens Biograph } \\
\quad 64\end{array}$ & $\mathrm{~N}$ & $\mathrm{~N}$ & $\begin{array}{l}\text { PFS - progression, death } \\
\text { related to lymphoma }\end{array}$ & $\begin{array}{l}\text { Median: } \\
\quad 34 \text { months }\end{array}$ & $\mathrm{N}$ & Moderate \\
\hline Zhou [49] & $\mathrm{N}$ & $\mathrm{N}$ & $\begin{array}{l}\text { GE Discovery ST } \\
\text { Siemens } \\
\text { Biograph } 64\end{array}$ & $\mathrm{~N}$ & $\mathrm{~N}$ & $\mathrm{~N}$ - not defined & $\begin{array}{l}\text { Median: } \\
30 \text { months }\end{array}$ & $\mathrm{N}$ & Moderate \\
\hline
\end{tabular}

PFS progressive free survival; $E F S$ event free survival; $O S$ overall survival; $F F P$ free from progression; $b w$ body weight; 1 Discovery $690, \mathrm{STE}, \mathrm{ST}$, RX, 600, 710, LS, Biograph HiRez, Truepoint, mCT, LSO, BGO and Gemini TF and GXL; EANM European Association of Nuclear Medicine

HR was 1.35 (CI 95\% 1.06-1.76) for PFS and 1.52 (CI $95 \%$ 1.15-2.02). However, there is considerable heterogeneity specifically in the PFS analysis $\left(\mathrm{I}^{2}=77 \%\right)$ and reporting bias is present because a number of studies which did not report any significance did not provide the results required to calculate a HR.

Of the studies which showed a prognostic ability for SUVmax, Gallicchio et al. reported this was the only imaging parameter able to predict PFS when compared to TLG and MTV in a small study of 52 DLBCL patients (26 early and 26 advanced stage) with a higher SUVmax associated with a longer PFS, the hazard ratio (HR) was $0.13(0.04-0.46)$ [25]. A study by Kwon et al. assessing 92 DLBCL (54 stage I/II, 38 stage II/IV) patients reported that a SUVmax of 10.5 was significant in predicting PFS, but this was not an independent prognostic predictor at multivariate analysis with clinical factors such as age, Lactate Dehydrogenase (LDH) level, stage, IPI score or Eastern Cooperative Oncology Group (ECOG) status [32]. Conversely, Miyazaki et al. demonstrated that SUVmax was an independent predictor of 3-year PFS and R-IPI [38]. Chang et al. found that tumour SUVmax $>19$ was a significant predictor of 3-year PFS, whereas the SUVmax of sternal uptake was an independent predictor of 3 -year OS in a study of 70 DLBCL patients [18]. The most extensive study evaluating SUVmax as a predictor of PFS and OS was performed by Ceriani et al. with a test cohort of 141 patients and a validation cohort of 113 patients, both containing a similar mix of stage and prognostic scores. SUVmax was not significant in predicting PFS or OS in either cohort [16]. b) $\mathrm{HL}$

Five studies have assessed the use of SUVmax as a predictive parameter in HL patients with only one reporting significance (Table 2). The largest by Akharti et al. showed no significant ability of SUVmax to predict PFS and OS in 267 stage I and II HL patients (74 early favourable) [12]. These findings were concordant with a study by Cottereau et al., who also found no significant ability of SUVmax to predict PFS or OS in 258 stage I and II patients. Angelopoulou et al. reported that SUVmax was a significant predictor of 5-year PFS in a study of 162 patients with a split of stages (stage I/II $=76$, stage III/IV = 86) [14]. The cohort was stratified into three risk groups, SUVmax <9, 9-18 and > 18 with five-year PFS rate being $93 \%, 81 \%$ and $58 \%$ respectively, multivariate analysis was not performed. Albano et al. studied the prognostic ability of liver to lesion SUV ratio and blood pool to lesion ratio in 123 older (age $>65$ years) HL patients [13]. They found that both parameters were significant (at univariate analysis) for PFS and OS. They also demonstrated these metrics to be independent prognostic markers when analysed with tumour stage, German Hodgkin Study Group (GHSG) risk group, MTV and TLG for PFS, and tumour stage, GHSG risk group and Deauville score for OS.

Factors affecting SUV such as scanner spatial resolution, image acquisition and PET reconstruction parameters combined with a relatively small number of events, variation in the number of early and advanced patients, differences in treatment and definition of PFS all influence the results [51, 


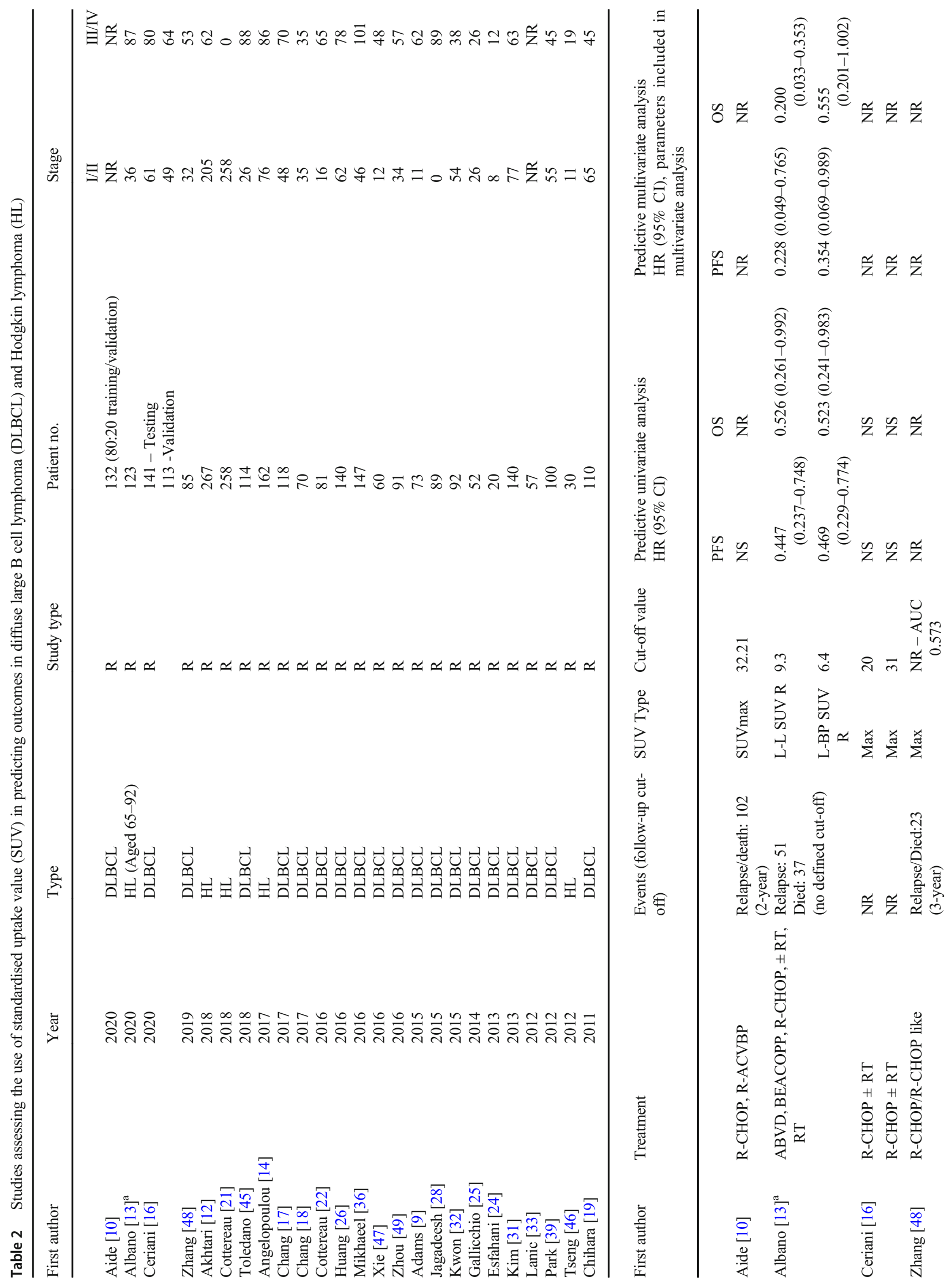




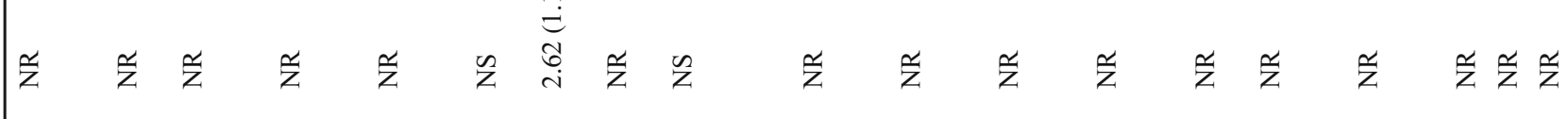

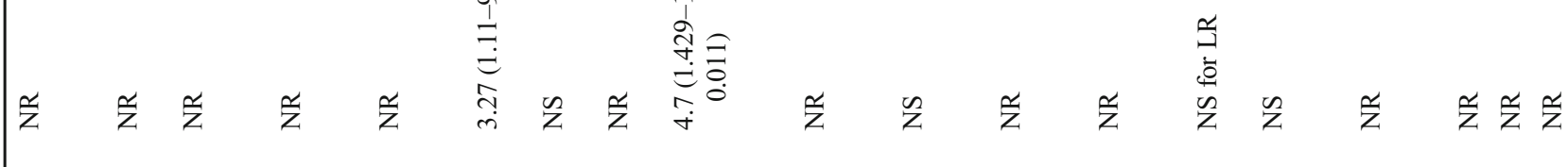

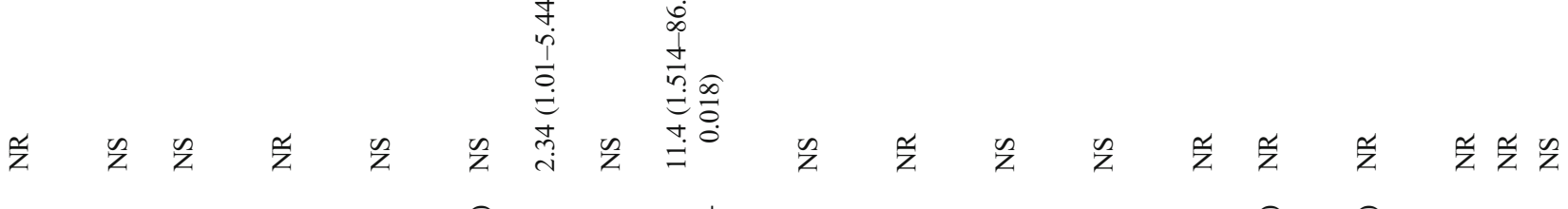

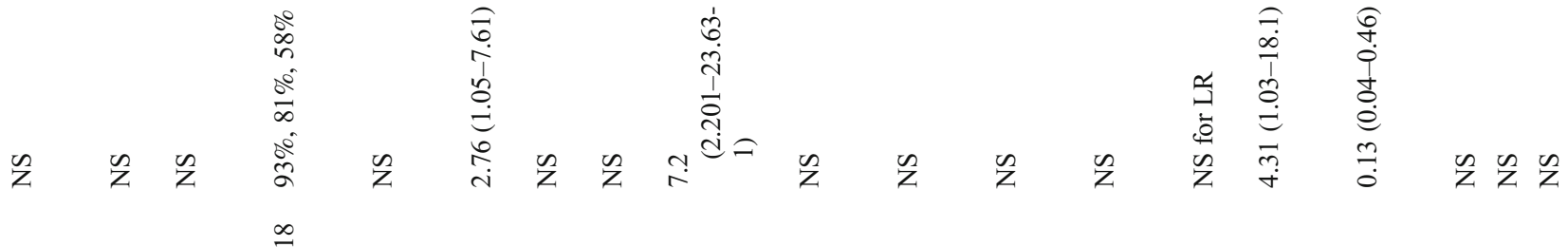

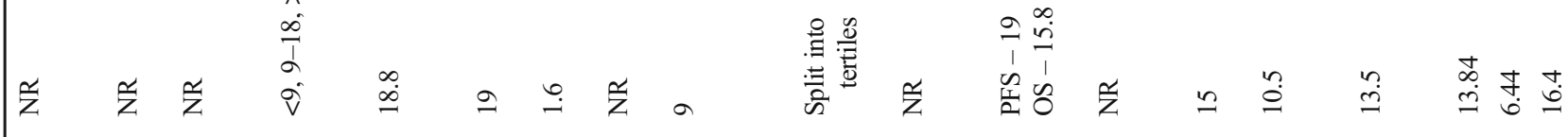

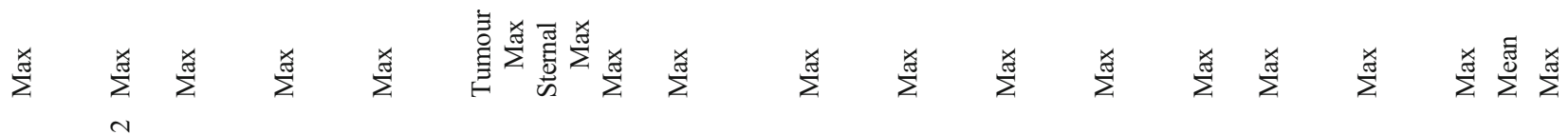

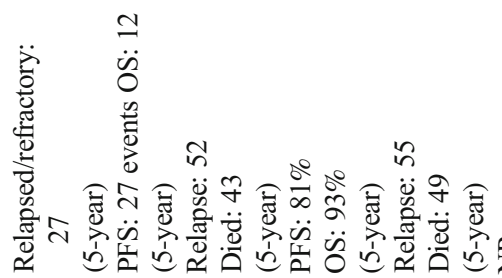

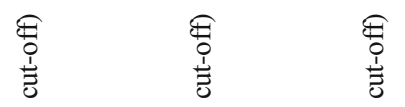

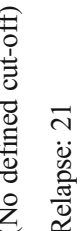

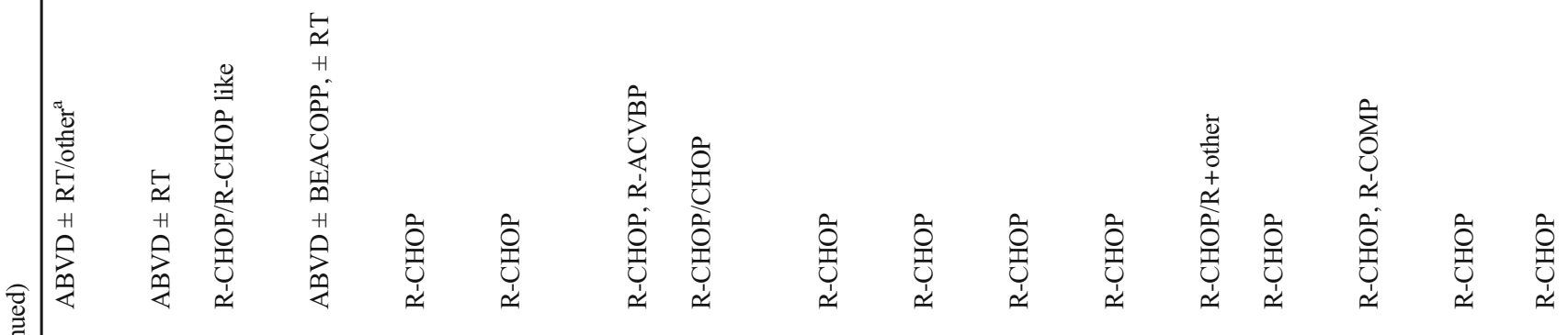

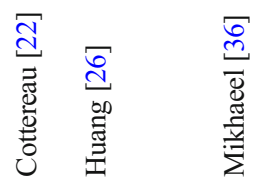

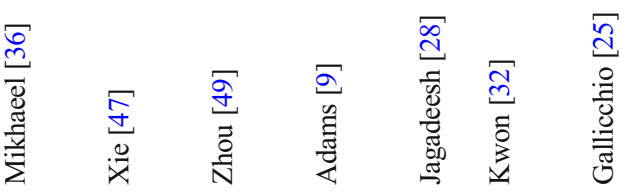

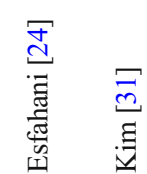




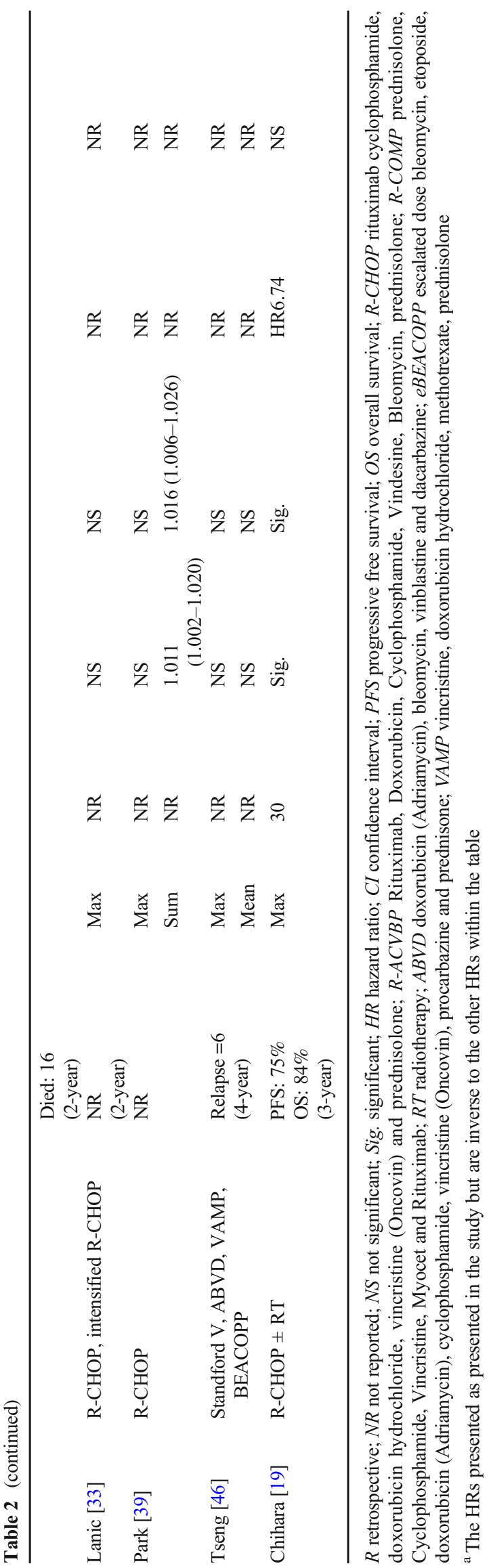

52]. This is reflected by the variation in cut-off/threshold values used to risk-stratify patients within each of the studies.

\section{Metabolic tumour volume and total lesion glycolysis for prediction of outcome}

\section{a) $\mathrm{DLBCL}$}

The potential utility of baseline MTV and TLG for predicting PFS and OS in patients with DLCBL has been reported in multiple studies (Table 3, Figs. 4 and 5). However, similar to SUVmax, there is heterogeneity in the cut-off values used which has led to variability in the reported survival rates between groups. Overall, the HR for MTV in PFS was 3.47 (CI 95\% 2.80-4.30) and 4.20 (CI 95\% 2.80-4.30) for OS. Again, reporting bias is present because a number of studies which did not report any significance did not provide the results required to calculate a HR.

One of the largest studies by Song et al. evaluated 169 patients with DLBCL (stage II and III without extranodal disease) treated with R-CHOP [44]. Patients with an MTV of $<220 \mathrm{~cm}^{3}$ had significantly better PFS and OS; 89.8 versus $55.6 \%$, and 93.2 versus $58.0 \%$, respectively [44]. MTV was predictive of PFS and OS regardless of stage. MTV remained significant when assessed using multivariate Cox regression with stage III disease, $\mathrm{HR}=5.30(95 \% 2.51-11.16)$ and $\mathrm{HR}=$ 7.01 (2.90-16.93) for 3-year PFS and 3-year OS, respectively. In another study, Song et al. reported that MTV was a prognostic predictor in 107 patients with bone marrow involvement (BMI); patients with an MTV of $>601.2 \mathrm{~cm}^{3}$ and BMI had worse PFS and OS survival compared to those with a smaller MTV and BMI [42]. Again, this was demonstrated to be an independent predictor when analysed with IPI, bulky disease, BMI, involved marrow MTV and $>2$ cytogenetic abnormalities with an $\mathrm{HR}=5.21$ (95\% CI 2.54-10.69) and $\mathrm{HR}=5.33$ (95\% CI 2.60-10.90) for PFS and OS, respectively. However, there was no significant difference in survival between the smaller MTV with BMI group and a comparison cohort of patients without BMI. MTV summarises disease burden; however, it does not account for spread. Cottereau et al. studied four different spatial metrics besides TLG and MTV in 95 DLBCL patients on baseline scans to determine if a predictive model could be created [20]. The spatial parameters consisted of Dmax (distance between two of the furthest lesions), Dmax bulk (distance between the largest lesion and furthest lesion away from this), SPREADbulk (sum of all distances between bulky lesions) and SPREAD (sum of all distances between lesions). They found that a model combining MTV and Dmax could significantly distinguish between three prognostic groups. The low-risk group with an MTV $<394 \mathrm{~cm}^{3}$ and a Dmax $<58 \mathrm{~cm}$ had a 4 -year PFS of $94 \%$ and OS of $97 \%$, the intermediate group with either an MTV 


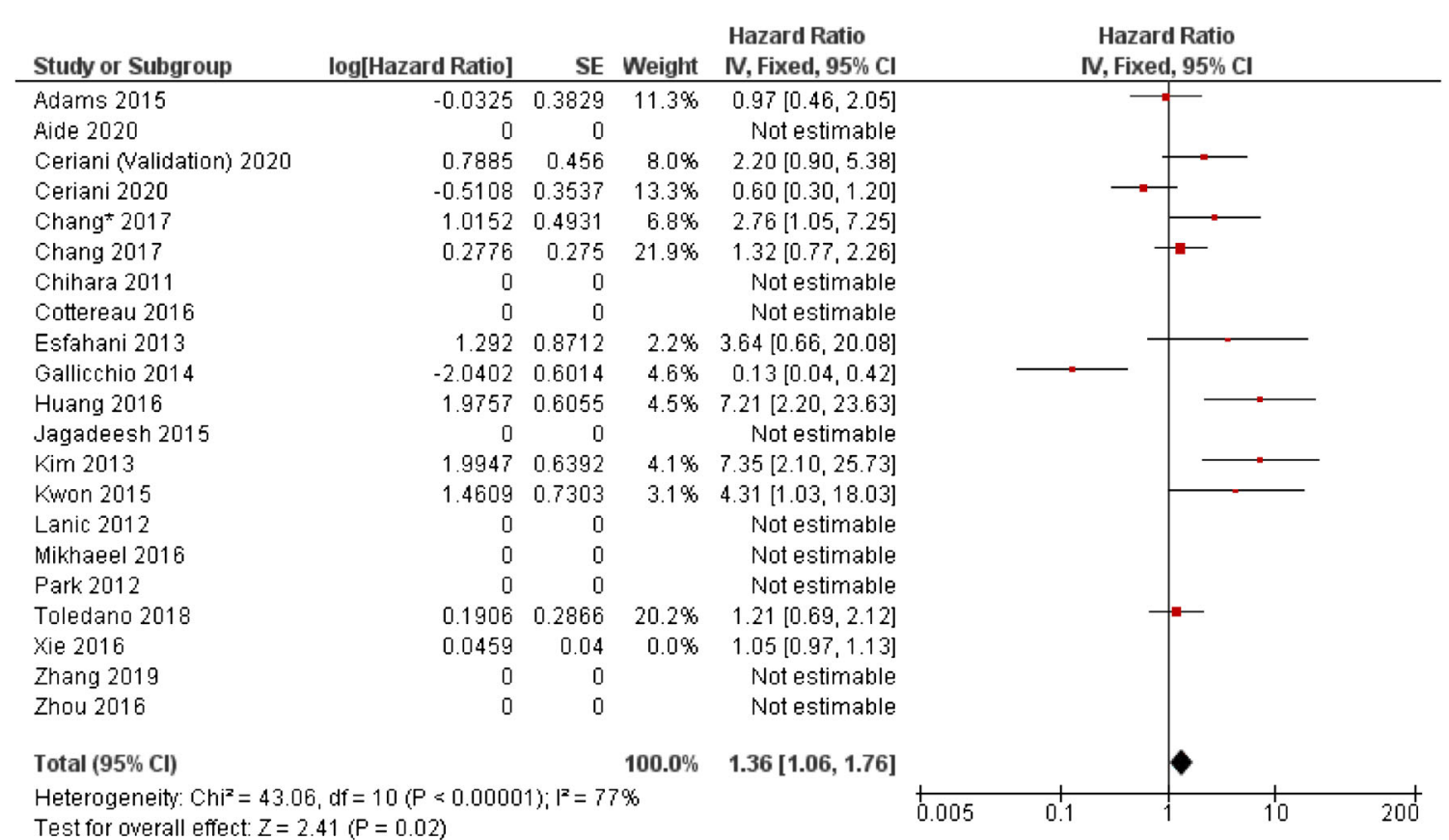

Fig. 2 Forest plot demonstrating hazard ratios for progression-free/event-free survival for patients with DLBCL using a dichotomous cut-off value derived from SUVmax. Studies which do not provide hazard ratios are included but no estimate is given

$>394 \mathrm{~cm}^{3}$ or a Dmax $>58 \mathrm{~cm}$ had a 4 -year PFS of $73 \%$ and OS of $88 \%$ and the high-risk group with a MTV $>394 \mathrm{~cm}^{3}$ and a Dmax $>58 \mathrm{~cm}$ had a 4-year PFS of $50 \%$ and OS of $53 \%$.

Zhou et al. reported that although high baseline MTV and TLG were associated with poorer prognosis, only TLG was an independent predictor of PFS and OS in a study of 91 patients [49]. In this study, patients who demonstrated complete or partial remission were more likely to relapse if they had a high baseline TLG (40 versus 9\%, $p=0.012$ ). A possible explanation for the discrepancy between the prognostic ability of MTV and TLG in this study may be related to the correlation between MTV and TLG, confounded by relatively small sample sizes. Kim et al. evaluated TLG calculated using different MTVs derived using 25, 50 and $75 \%$ SUVmax thresholds in a mixed cohort $(n=140)$ of early and advanced stage DLBCL patients being treated with R-CHOP [31]. They found that all

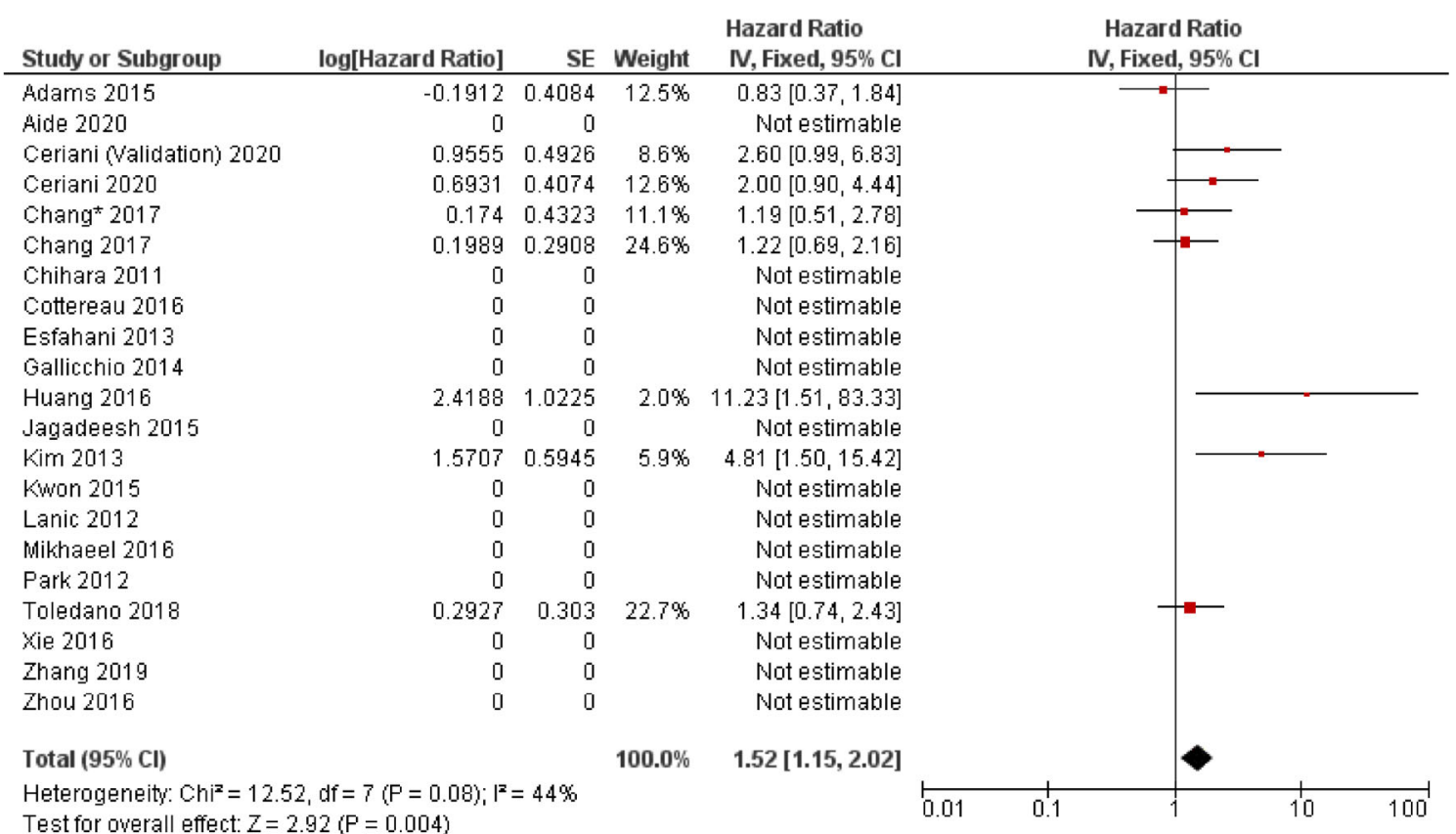

Fig. 3 Forest plot demonstrating hazard ratios for overall survival for patients with DLBCL using a dichotomous cut-off value derived from the SUVmax. Studies which do not provide hazard ratios are included but no estimate is given 


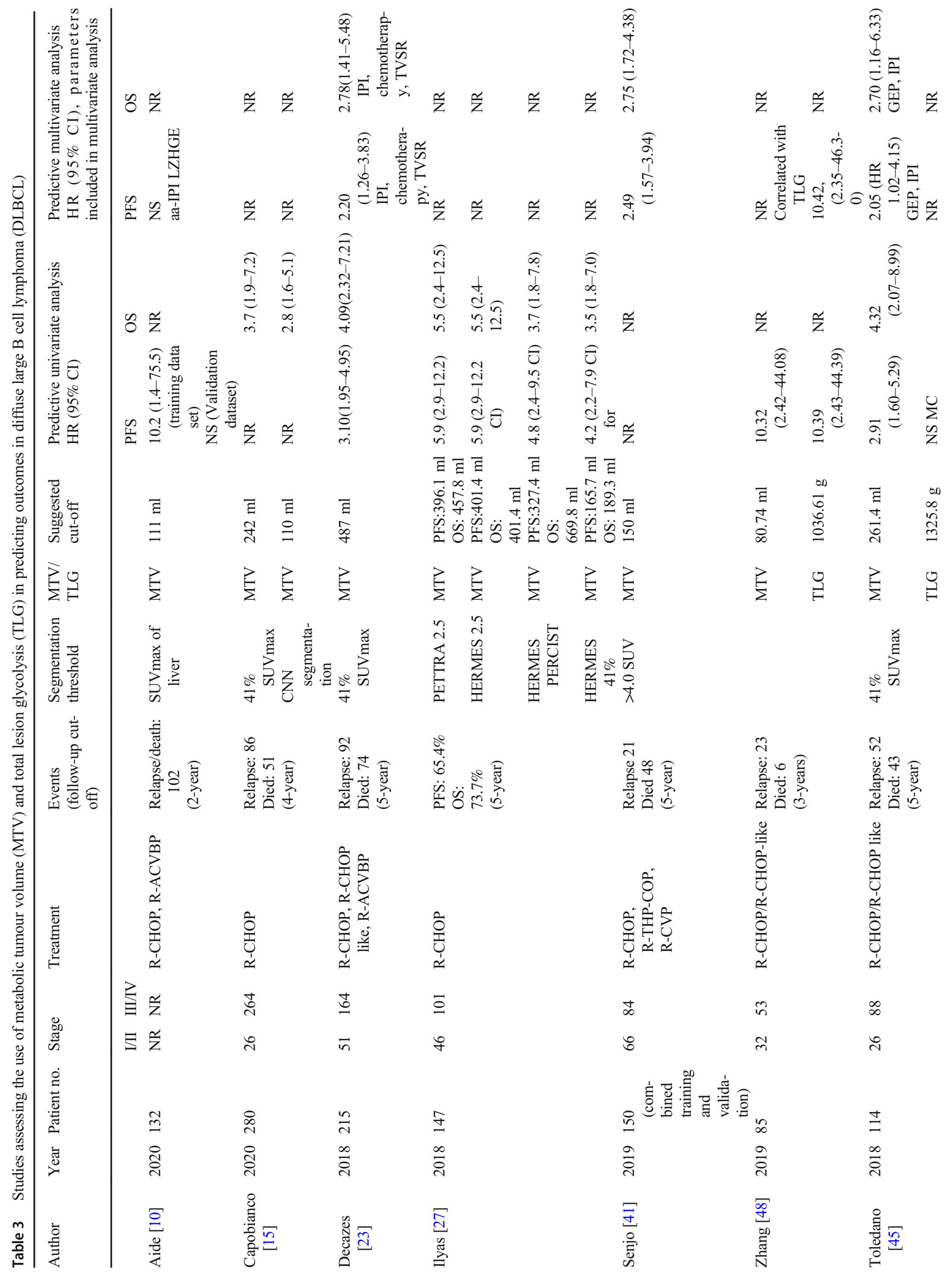




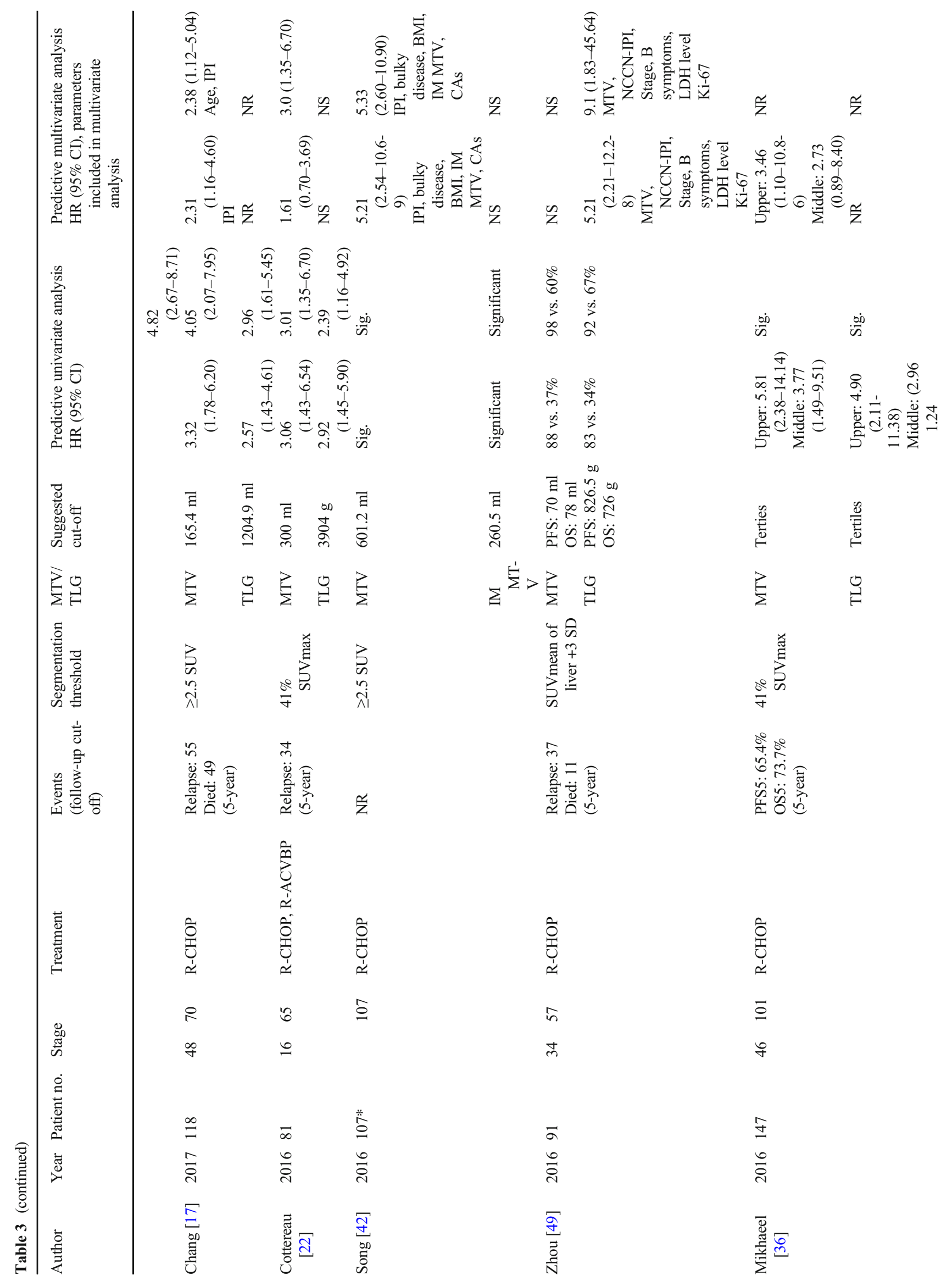




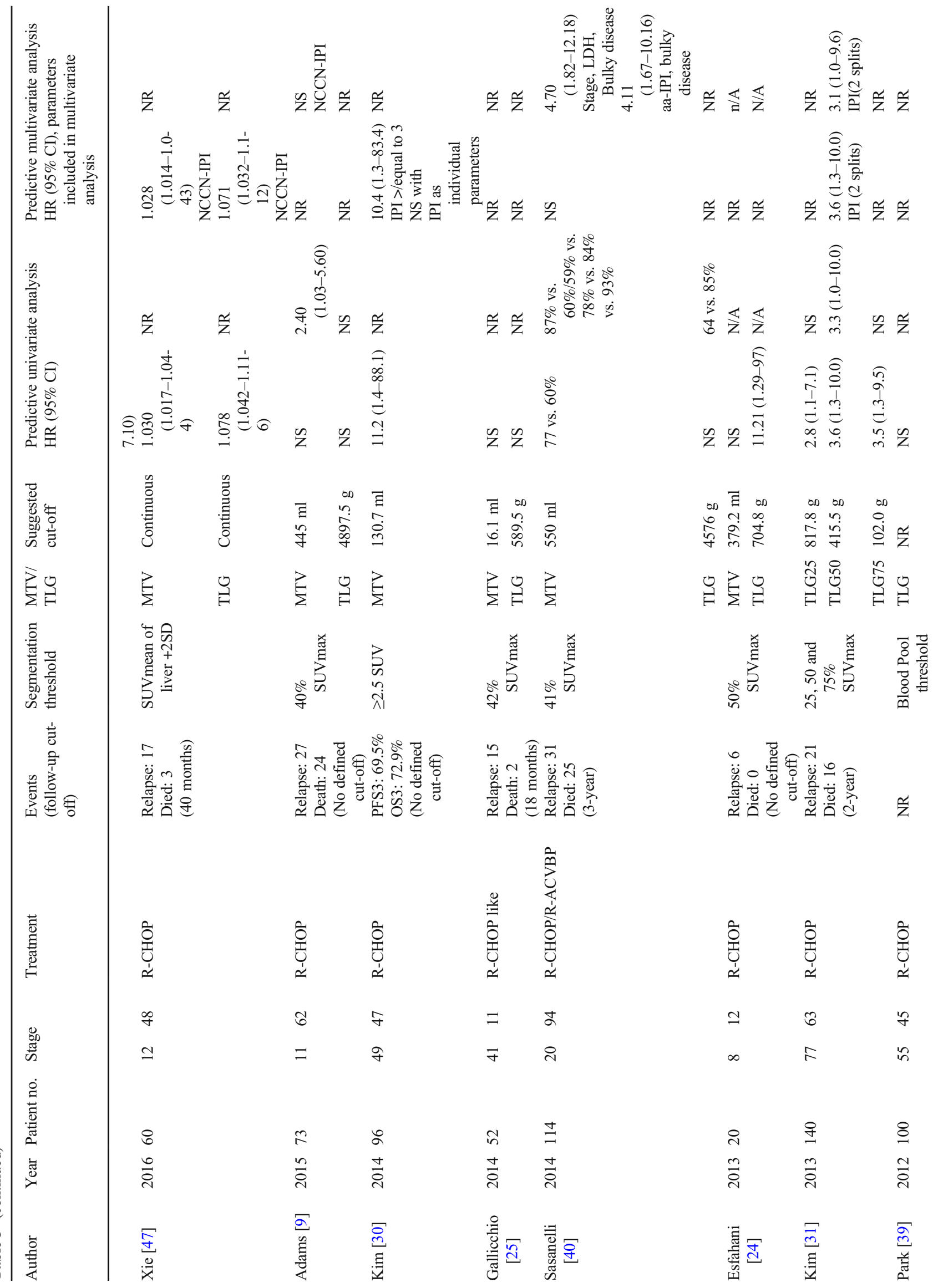


methods for calculating TLG were predictive of 2-year PFS, but only TLG50 was predictive of 2-year OS. Ilyas et al. also studied variation in segmentation technique and its potential to impact on predicting outcome in 147 DLBCL patients (46 stage I/II, 101 stage III/IV) all treated with R-CHOP [27]. The four segmentation techniques consisted of a threshold of SUV 2.5 on two software packages (PETTRA and Hermes), $41 \%$ SUVmax on Hermes software and an uptake higher than SUVmean of a $3-\mathrm{cm}^{3}$ region of interest (ROI) within the right lobe of the liver (PERCIST) using the Hermes software. They found a strong agreement between all four methods, with the lowest intraclass coefficient being between PERCIST and $41 \%$ SUVmax thresholds being 0.86 . They also reported similar receiver operator curves (ROC) between the four methods with the area under the curve (AUC) ranging from 0.74 to 0.76 for PFS, and 0.71 to 0.75 for OS. All four methods were significant predictors of PFS and OS. However, as stated in the paper, no method is likely to apply to all patients generally. Large heterogeneous masses are likely to be undersized with percentage thresholds, low uptake lesions may be missed using a standard threshold method and disease involving the liver may impede its use as the background value. This may have a more significant impact when further metrics are introduced, such as those based on texture when the size of the contour can also influence the reported values. The segmentation technique of choice also needs to be easily replicated. Recently, Capobianco et al. assessed the use of artificial intelligence (AI) using a convolutional neural network (CNN) to segment the MTV [15]. They found that AI-derived MTV correlated with reference MTV derived by two independent readers with a classification accuracy of $85 \%$. Automatic segmentation is a key step required to enable implementation of MTV or TLG into clinical practice.

\section{HL}

Fewer studies have investigated the predictive ability of MTV and TLG in HL patients than in DLBCL (Table 4, Figs. 6 and 7). This is likely due to the higher survival rate of HL limiting the number of events demonstrated in a single centre and the variation in treatments and scoring systems for a favourable and unfavourable disease, which affect multi-centre studies. The majority of studies involved patients on an adaptive ABVD treatment regime, and results may not be transferrable to patients being treated with an adaptive BEACOPP regime. This confounding issue was highlighted in a study by Mettler et al. who assessed the prognostic ability of MTV in 310 patients with advanced HL being treated with eBEACOPP using four different contouring methods involving summation of the volume of each disease site using different defined thresholds: $41 \%$ SUVmax of each disease site, a threshold of liver SUVmax, a threshold of liver SUVmean and a fixed threshold of 2.5 SUV [35]. They found that MTV was 


$\begin{array}{lrrrrr} & & & \text { Hazard Ratio } \\ \text { Study or Subgroup } & \text { log[Hazard Ratio] } & \text { SE } & \text { Weight } & \text { IV, Fixed, 95\% Cl } & \text { Hazard Ratio } \\ \text { IV, Fixed, 95\% Cl }\end{array}$

Fig. 4 Forest plot demonstrating hazard ratios for progression-free survival for patients with DLBCL using a dichotomous cut-off value derived from the metabolic tumour volume. Studies which do not provide hazard ratios are included but no estimate is given

predictive of interim PET response regardless of segmentation methodology; however, none was able to predict OS and PFS reliably. The divergent findings compared to previous studies are likely related to low event numbers and using a different treatment regime. Albano et al. demonstrated the significant ability of both MTV and TLG derived from $41 \%$ SUVmax in predicting PFS in both univariate and multivariate analysis in a cohort of 123 elderly patients with a mix of different treatment regimens. However, neither TLG nor MTV were predictive of OS. Cottereau et al. and Akhtari et al. both assessed the ability of MTV in cohorts of patients consisting of stage I and II disease $[12,21]$. Cottereau et al. found that MTV derived from $>2.5$ SUV was significant in predicting 5-year PFS and OS and was significant in multivariate analysis when assessed with different early disease scoring systems. Akhtari et al. found that MTV and TLG derived from $>2.5$ SUV thresholding and manual soft tissue contouring were significant predictors of 5-year PFS. Reporting bias is present because a number of studies which did not report any significance did not provide the results required to calculate a HR.

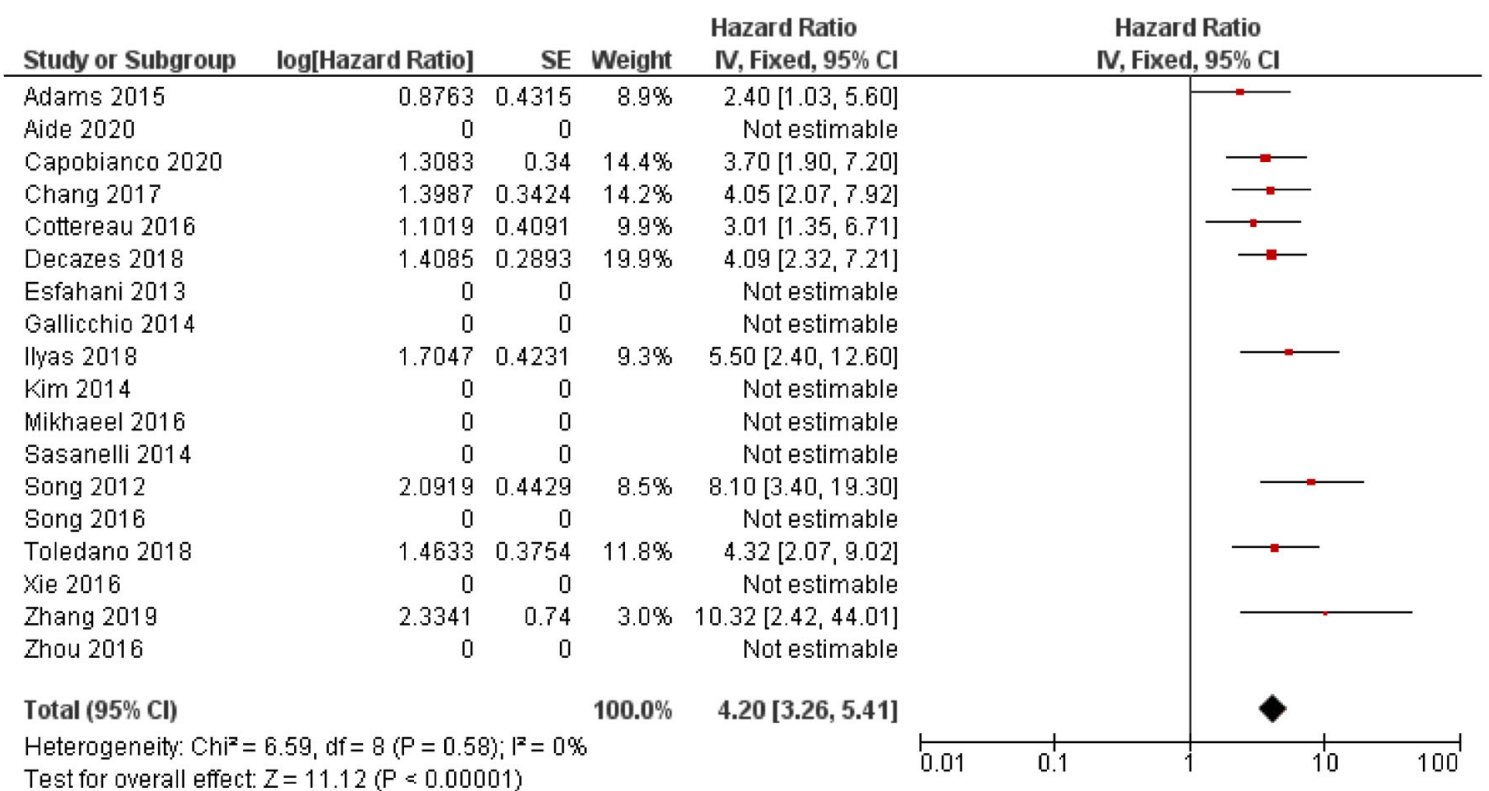

Fig. 5 Forest plot demonstrating hazard ratios for overall survival for patients with DLBCL using a dichotomous cut-off value derived from the metabolic tumour volume. Studies which do not provide hazard ratios are included but no estimate is given 


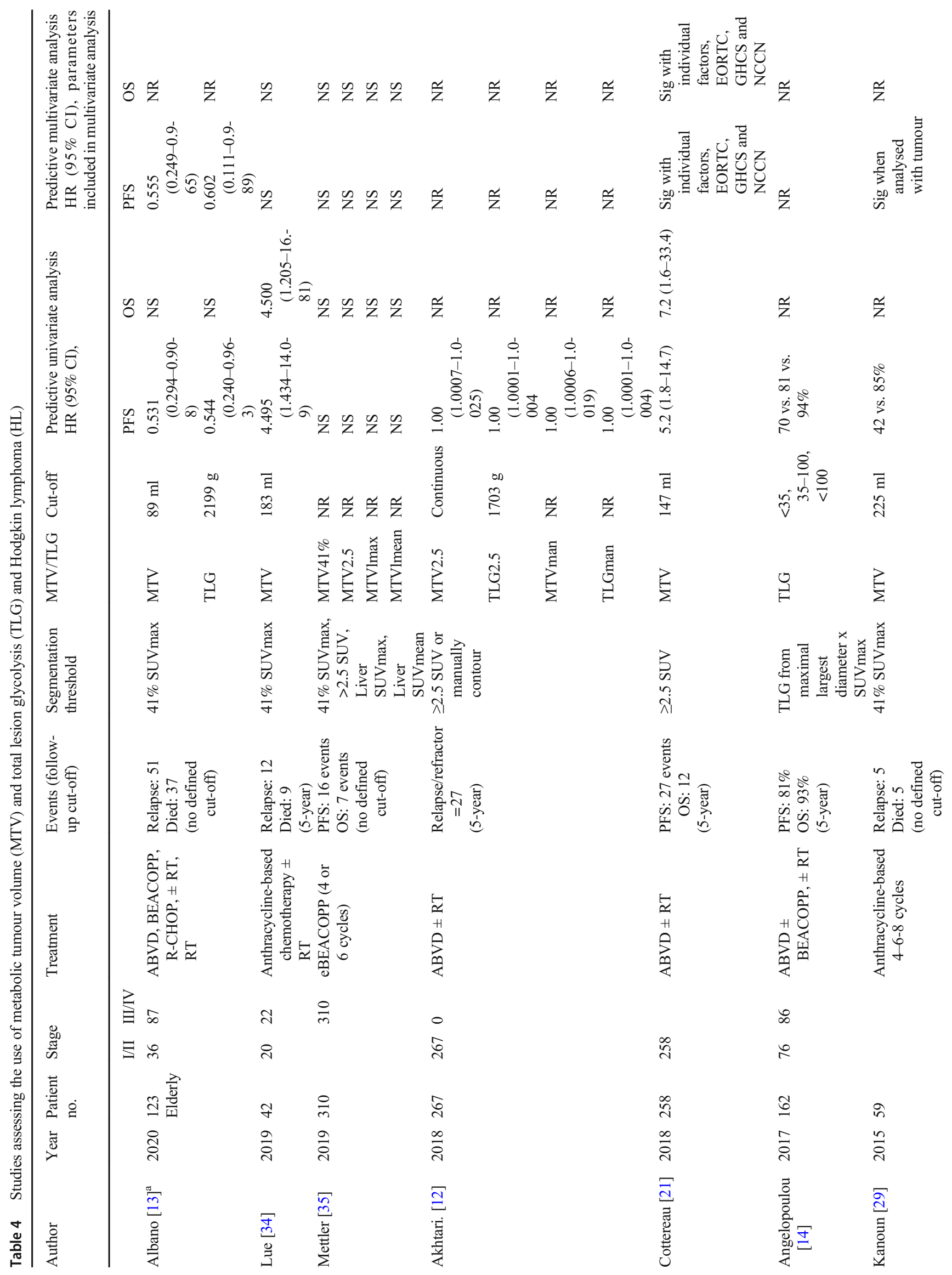




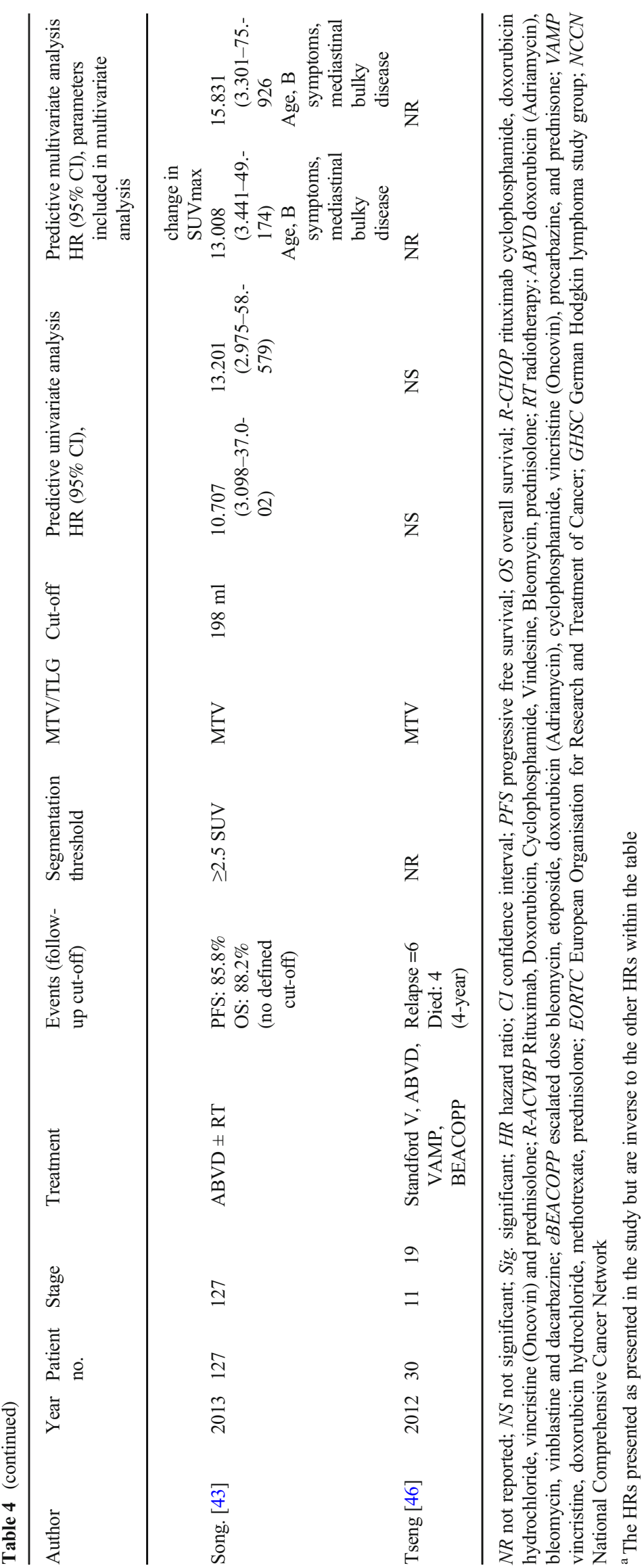




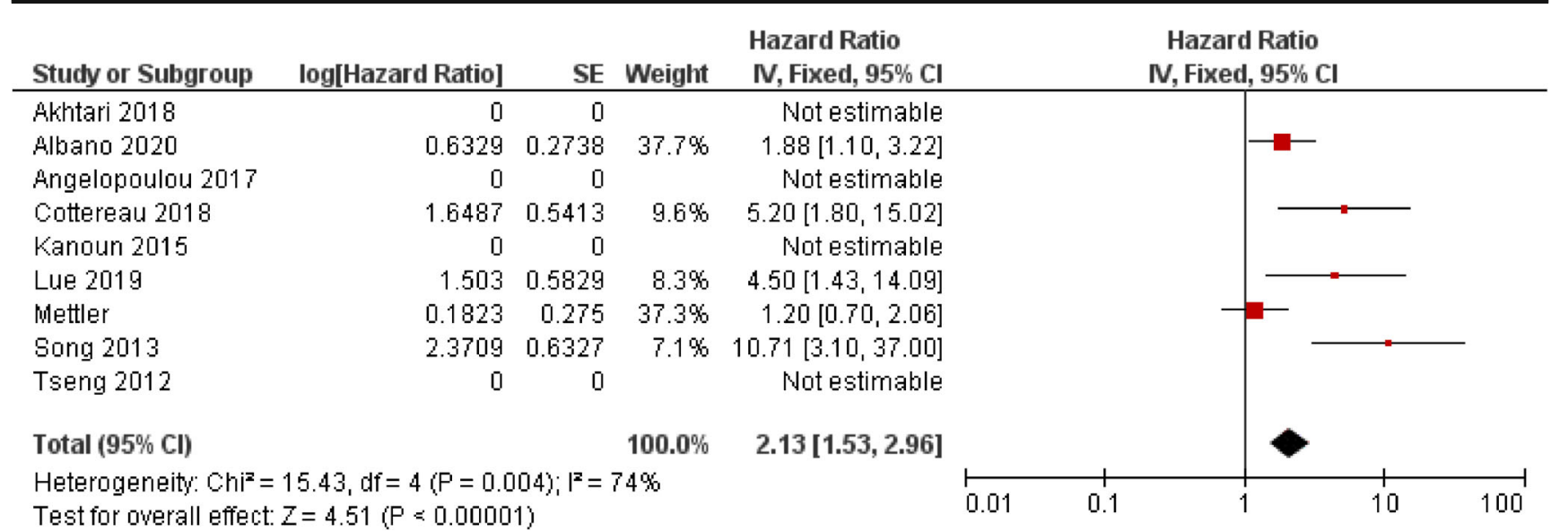

Fig. 6 Forest plot demonstrating hazard ratios for progression-free survival for patients with HL using a dichotomous cut-off value derived from the metabolic tumour volume. Studies which do not provide hazard ratios are included but no estimate is given

The overall HR for MTV in PFS was 2.13 (CI 95\% 1.53-2.96) and $2.13(1.43-3.16)$ in OS. Both were associated with high levels of heterogeneity, $I^{2}=74 \%$ for PFS and $I^{2}=70 \%$ for OS.

Similar to DLBCL, clinical implementation of MTV and TLG in HL depends on reaching a consensus regarding segmentation methodology, each giving different variations in the volumes measured and will be facilitated by an automated process. However, variation in treatment is likely also to play an impact, and this aspect needs assessing in larger multicentre studies.

\section{Textural and shape analysis for outcome prediction}

Textural analysis or radiomics relates to transformation of images into mineable high-dimensional data permitting invisible feature extraction, analysis and modelling for noninvasive phenotyping and outcome prediction [53]. Radiomic features can be studied in isolation or increasingly are being combined with clinical and genomic features as part of the rapidly expanding field of integrated diagnostics [54].

Aide et al. studied the use of PET/CT-derived textural features, clinical and imaging parameters to predict 2-year PFS in DLBCL patients [10]. They split patients into training $(n=$ $105)$ and validation sets $(n=27)$ and found that Long-Zone High-Grey Level Emphasis (LZHGE) was the only independent predictor when analysed with IPI and MTV. On the validation set, it was found that a high LZHGE $>1,264,925.92$ was associated with a 2-year PFS of $60 \%$ whereas patients with a low LZGHE had a PFS of $94.1 \%$. The study has some limitations as only the largest area of disease was analysed, a breakdown of disease stage was not presented and 14 patients did not have standard (R-CHOP) therapy. Another study by Aide et al. investigated the diagnostic and prognostic value of axial skeletal textural features derived from PET/CT in patients with DLBCL in a retrospective cohort of 82 patients [11]. The CT dataset was initially contoured using a segmentation threshold of $>150$ Hounsfield units (HU) with the spinal column and half of the pelvis included. They reported that the first-order parameter skewness had the highest AUC for

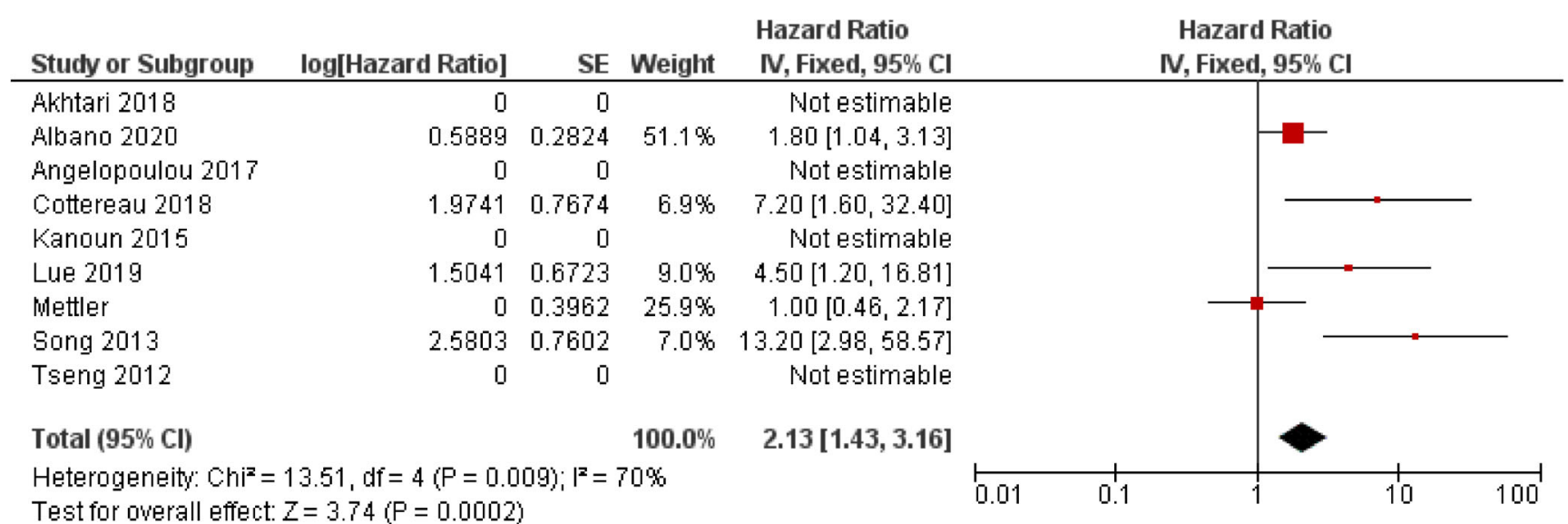

Fig. 7 Forest plot demonstrating hazard ratios for overall survival for patients with HL using a dichotomous cut-off value derived from the metabolic tumour volume. Studies which do not provide hazard ratios are included but no estimate is given 
Fig. 8 Select axial $(\mathbf{a}-\mathbf{c})$ and coronal slices (d) from an FDG PET/CT study from a patient with DLBCL demonstrating three different contouring methods (green $=41 \%$ SUVmax; red $=1.5$ $\mathrm{x}$ SUVmean of the liver; purple $=$ 4.0 SUV). For smaller lesions, the $41 \%$ SUVmax contour is larger than the other two methods, black arrow and arrowhead. For larger more heterogenous lesions, the $41 \%$ SUVmax is the smallest of the three contours (blue arrow)

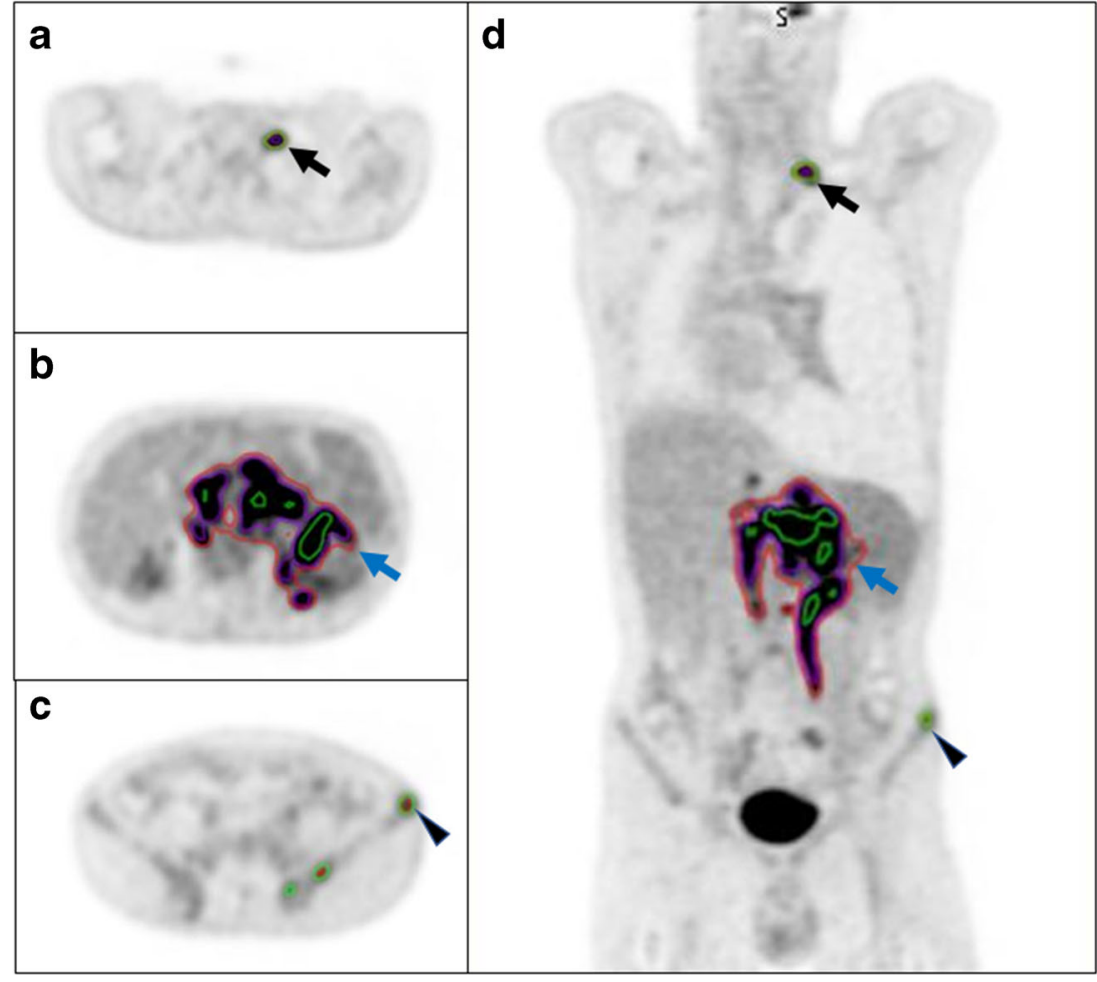

predicting BMI and that a cut-off value of 1.26 produced a sensitivity, specificity, PPV and NPV of 82, 82, 62 and 93\%, respectively. In addition, a skewness value of $<1.26$ was associated with a greater 2-year PFS and OS. This was true even for 60 patients without BMI. The study had a low event rate (22 patients had BMI), which limits the ability to create a robust prognostic model.

Lue et al. investigated the use of 11 first-order, 39 higherorder features and 400 wavelet features for predicting PFS and OS in $42 \mathrm{HL}$ patients (20 stage I/II, 22 stage III/IV) with 21 events within the cohort (12 relapses, 9 deaths) [34]. They found 173 radiomic features, which were significant predictors of progression after correction for multiple testing. To avoid multicollinearity, they only selected the top two features according to the AUC from each group to be included in the univariate and multivariate analysis. MTV was selected based on previous studies. They demonstrated that SUV kurtosis, stage and intensity non-uniformity (INU) derived from Grey-Level Run Length Matrix (GLRLM) were independent predictors of PFS and only disease stage and INU derived from GLRLM were independent predictors of OS.

Decazes et al. retrospectively studied PET/CT scans of 215 DLBCL patients to assess the utility of total tumour surface (TTS) and tumour volume surface ratio (TVSR) as predictive biomarkers [23], TVSR being the ratio between MTV and TTS. MTV had the highest AUC for both OS and PFS (0.71 and 0.67$)$ when compared to TTS (0.69 and 0.66) and TVSR (0.65 and 0.61) [23]. It was reported that TVSR, MTV, IPI and type of chemotherapy were all independent prognostic parameters. Milogrom et al. investigated the use of a support
Table 5 Limitations of the current literature and opportunities for future work

\begin{tabular}{ll}
\hline Limitation & Opportunity \\
\hline $\begin{array}{l}\text { 1. Relatively small retrospective } \\
\text { cohorts with limited events }\end{array}$ & Establishing multi-centre networks for future larger-scale studies \\
$\begin{array}{l}\text { 2. Multiple segmentation techniques } \\
\text { used }\end{array}$ & $\begin{array}{c}\text { Consensus on segmentation technique for MTV and TLG and } \\
\text { development of automated AI-methods which are implemented } \\
\text { within reporting software by manufacturers }\end{array}$ \\
$\begin{array}{l}\text { 3. Single site models using a single } \\
\text { dataset }\end{array}$ & $\begin{array}{c}\text { Internal and external validation should be routinely performed and } \\
\text { facilitated by networks }\end{array}$ \\
$\begin{array}{l}\text { 4. Varying predictive end points } \\
\begin{array}{l}\text { Consensus on clinically relevant predictors } \\
\text { nonall numbers of papers using }\end{array}\end{array}$ & $\begin{array}{c}\text { Using different machine learning and deep learning models to aid in } \\
\text { imaging analysis and outcome prediction }\end{array}$ \\
\hline
\end{tabular}


vector machine model based on first and second-order radiomic features derived from baseline PET/CT to predict relapse or refractory disease in 167 stage I-II HL patients with mediastinal involvement [37]. Ten of the groups formed the training set, and two were designated the validation set with each group containing a single event $(n=12)$. Five features were selected as the most predictive (SUVmax, MTV, InformationMeasureCorr1, InformationMeasureCorr2 and InverseVariance derived from GLCM 2.5). InformationMeasureCorr1 and InformationMeasureCorr 2 are the first and second measures of theoretic correlation and Inverse-Variance is weighting of random variables to minimise variance. By combining these features, the AUC for predicting relapse for patients with mediastinal disease was 0.95 . This outperformed TLG and MTV. This work highlights the potential for using AI-methods in lymphoma assessment. However, the study is limited to HL with mediastinal involvement with again small numbers of events.

Senjo et al. demonstrated that a high metabolic heterogeneity $(\mathrm{MH})$ was a predictor of 5-year PFS and OS in DLBCL across both training $(n=86)$ and validation cohorts $(n=64)$ treated at two centres [41]. They found that $\mathrm{MH}$ remained a significant predictor for 5-year OS for both cohorts when analysed in multivariate analysis with an ECOG score of $>2$, and an LDH with a relative risk of 4.75 (95\% CI 1.25-18.1) and relative risk of $4.92(95 \%$ CI 1.09-17.03) in the training and validation groups, respectively. A model was created which combined MH and MTV, which successfully risk stratified the combined training and validation cohorts into three risk groups: low MH and low MTV, low MH and high MTV or high MH and low MTV, and high MH and high MTV, with the 5 -year OS being 90.4 vs. 69.5 vs. $34.8 \%$, respectively; $P<0.001$ and 5 -year PFS, 84.1 vs. 43.6 vs. $27.0 \%, P<$ 0.001 respectively.

\section{Current limitations and future challenges}

One issue needing to be addressed when using imaging parameters derived from PET for predictive modelling is the relatively low spatial resolution, which influences how much of the avidity is included within a volume when different thresholding techniques are utilised (Fig. 8) [55]. Meignan et al. used a phantom model to validate their MTV thresholding method for a patient cohort [56]. They found that a 41\% SUVmax threshold gave the best concordance between contoured and actual volumes. $41 \%$ SUVmax thresholding also gave the best agreement between reviewers using the Lin concordance correlation coefficient $(\mathrm{pc})(\rho \mathrm{c}=0.986$, CI 0.97-0.99). However, for successful clinical implementation, the time it takes to implement as well as the accuracy of the thresholding method needs be considered. The use of a semi- automated method such as the one reported by Burggraaff et al. [57] or a deep learning derived volume as reported by Capobianco et al. is required [15]. Predictive models also need to be tested and adapted for new treatments or histological markers [58]. The ability to be able to predict worse outcomes could allow for future treatment stratification. There is an area of unmet need with few active studies at present. There are currently only two open/recruiting studies listed on clinicaltrials.gov assessing PET/CT parameters for outcome prediction in DLBCL, and no registered studies assessing outcomes in HL patients.

Other important limitations of the published work highlighted in this systematic review are variability in methodology and lack of external validation (Table 5). This presents a number of opportunities for the future (Table 5). Further study into the use of AI for imaging-based outcome prediction in lymphoma which may permit more accurate prediction of prognosis/treatment outcome is needed. This might also facilitate more efficient image analysis and actionable clinical decision support potentially guiding tailored treatment for individual patients. However, there is the requirement for large volumes of data necessary to train algorithms which can then be vigorously validated for reproducibility and generalizability which will require crossinstitutional collaboration via imaging networks to support the establishment of multi-centre trials. Implementation studies and health economic research will also be critical for clinical adoption by demonstrating that any AI application is reliable and value-based.

All the described limitations have led to a medium and high risk of bias within the literature as evaluated with our QUIPS tool. The decision to retain papers with a high risk of bias was taken as it was felt that this itself would introduce bias into the review. However, this does mean the results need to be interpretated with caution. Further work in this area is clearly warranted and efforts should be made when designing future studies to carefully consider the methodology employed so as to minimise the risk of bias which is prevalent in this field of work to date.

\section{Conclusion}

Multiple reports suggest the potential utility of various PET/ CT-derived imaging parameters in lymphoma outcome modelling. Most studies are retrospective and lack external validation of described models. Robustness across different scanning protocols and institutions has also not been verified, and clinical implementation remains a future aspiration. AI techniques may offer a potential solution to some limitations of predictive modelling in this clinical scenario and warrant further evaluation. 
Supplementary Information The online version contains supplementary material available at https://doi.org/10.1007/s00259-021-05233-2.

Funding Open access funding provided by University of Leeds. R Frood, F Gleeson, C Patel and A Scarsbrook received funding from Innovate UK. The funders had no role in study design, data collection and analysis, decision to publish or preparation of the manuscript.

\section{Declarations}

Conflict of interest R Frood, F Gleeson, C Patel and A Scarsbrook received funding from Innovate $\mathrm{UK}$; the funders had no role in study design, data collection or analysis. C Burton, C Tsoupmas and A Frangi have no conflict of interest.

Ethical approval This article does not contain any studies with human participants or animals performed by any of the authors.

Open Access This article is licensed under a Creative Commons Attribution 4.0 International License, which permits use, sharing, adaptation, distribution and reproduction in any medium or format, as long as you give appropriate credit to the original author(s) and the source, provide a link to the Creative Commons licence, and indicate if changes were made. The images or other third party material in this article are included in the article's Creative Commons licence, unless indicated otherwise in a credit line to the material. If material is not included in the article's Creative Commons licence and your intended use is not permitted by statutory regulation or exceeds the permitted use, you will need to obtain permission directly from the copyright holder. To view a copy of this licence, visit http://creativecommons.org/licenses/by/4.0/.

\section{References}

1. Shanbhag S, Ambinder RF. Hodgkin lymphoma: a review and update on recent progress. CA Cancer J Clin. 2018;68:116-32.

2. SEER. SEER Cancer Statistics Review. 1975-2016. 2018.

3. Armitage JO, Gascoyne RD, Lunning MA, Cavalli F. NonHodgkin lymphoma. Lancet. 2017;390:298-310.

4. Horvat M, Zadnik V, Šetina TJ, Boltežar L, Goličnik JP, Novaković $\mathrm{S}$, et al. Diffuse large B-cell lymphoma: 10 years' real-world clinical experience with rituximab plus cyclophosphamide, doxorubicin, vincristine and prednisolone. Oncol Lett. 2018;15:3602-9.

5. El-Galaly TC, Gormsen LC, Hutchings M. PET/CT for staging; past, present, and future. Semin Nucl Med. 2018;48:4-16.

6. Liberati A, Altman DG, Tetzlaff J, Mulrow C, Gøtzsche PC, Ioannidis JPA, et al. The PRISMA statement for reporting systematic reviews and meta-analyses of studies that evaluate health care interventions: explanation and elaboration. J Clin Epidemiol. 2009;62(10):e1-e34.

7. Hayden JA, van der Windt DA, Cartwright JL, Co P. Research and reporting methods annals of internal medicine assessing bias in studies of prognostic factors. Ann Intern Med. 2013;158:280-6.

8. Grooten WJA, Tseli E, Äng BO, Boersma K, Stålnacke B-M, Gerdle B, et al. Elaborating on the assessment of the risk of bias in prognostic studies in pain rehabilitation using QUIPS - aspects of interrater agreement. Diagn Progn Res. 2019;3:1-11.

9. Adams HJA, de Klerk JMH, Fijnheer R, Heggelman BGF, Dubois SV, Nievelstein RAJ, et al. Prognostic superiority of the National Comprehensive Cancer Network International Prognostic Index over pretreatment whole-body volumetric-metabolic FDG-PET/CT metrics in diffuse large B-cell lymphoma. Eur J Haematol. 2015;94:532-9.
10. Aide N, Fruchart C, Nganoa C, Gac A, Lasnon C. Baseline 18 FFDG PET radiomic features as predictors of 2-year event-free survival in diffuse large $B$ cell lymphomas treated with immunochemotherapy. Eur Radiol. 2020;30(8):4623-32.

11. Aide N, Talbot M, Fruchart C, Damaj G, Lasnon C. Diagnostic and prognostic value of baseline FDG PET/CT skeletal textural features in diffuse large B cell lymphoma. Eur J Nucl Med Mol Imaging. 2018:45:699-711.

12. Akhtari M, Milgrom SA, Pinnix CC, Reddy JP, Dong W, Smith GL, et al. Reclassifying patients with early-stage Hodgkin lymphoma based on functional radiographic markers at presentation. Blood. 2018;131:84-94.

13. Albano D, Mazzoletti A, Spallino M, Muzi C, Zilioli VR, Pagani C, et al. Prognostic role of baseline 18F-FDG PET/CT metabolic parameters in elderly HL: a two-center experience in 123 patients. Ann Hematol. 2020;99:1321-30.

14. Angelopoulou MK, Mosa E, Pangalis GA, Rondogianni P, Chatziioannou S, Prassopoulos V, et al. The significance of PET/ $\mathrm{CT}$ in the initial staging of Hodgkin lymphoma: experience outside clinical trials. Anticancer Res. 2017;37:5727-36.

15. Capobianco N, Meignan MA, Cottereau A-S, Vercellino L, Sibille L, Spottiswoode B, et al. Deep learning FDG uptake classification enables total metabolic tumor volume estimation in diffuse large Bcell lymphoma. J Nucl Med. 2020;62:30-36.

16. Ceriani L, Gritti G, Cascione L, Pirosa MC, Polino A, Ruberto T, et al. SAKK38/07 study: integration of baseline metabolic heterogeneity and metabolic tumor volume in DLBCL prognostic model. Blood Adv. 2020;4:1082-92.

17. Chang C-C, Cho S-F, Chuang Y-W, Lin C-Y, Chang S-M, Hsu W-L, et al. Prognostic significance of total metabolic tumor volume on $18 \mathrm{~F}$-fluorodeoxyglucose positron emission tomography/ computed tomography in patients with diffuse large B-cell lymphoma receiving rituximab-containing chemotherapy. Oncotarget. 2017;8:99587-600.

18. Chang $\mathrm{C}, \mathrm{Cho} \mathrm{S}, \mathrm{Tu} \mathrm{H}$, Lin $\mathrm{C}$, Chuang Y, Chang S, et al. Tumor and bone marrow uptakes on $[18 \mathrm{~F}]$ fluorodeoxyglucose positron emission tomography/computed tomography predict prognosis in patients with diffuse large B-cell lymphoma receiving rituximabcontaining chemotherapy. Med. 2017;96(45):e8655.

19. Chihara D, Oki Y, Onoda H, Taji H, Yamamoto K, Tamaki T, et al. High maximum standard uptake value (SUVmax) on PET scan is associated with shorter survival in patients with diffuse large B cell lymphoma. Int J Hematol. 2011;93:502-8.

20. Cottereau AS, Nioche C, Dirand AS, Clerc J, Morschhauser F, Casasnovas O, et al. 18F-FDG PET dissemination features in diffuse large B-cell lymphoma are predictive of outcome. J Nucl Med. 2020;61:40-5.

21. Cottereau AS, Versari A, Loft A, Casasnovas O, Bellei M, Ricci R, et al. Prognostic value of baseline metabolic tumor volume in earlystage Hodgkin lymphoma in the standard arm of the H10 trial. Blood. 2018;131:1456-63.

22. Cottereau AS, Lanic H, Mareschal S, Meignan M, Vera P, Tilly H, et al. Molecular profile and FDG-PET/CT Total metabolic tumor volume improve risk classification at diagnosis for patients with diffuse large B-cell lymphoma. Clin Cancer Res. 2016;22:3801-9.

23. Decazes P, Becker S, Toledano MN, Vera P, Desbordes P, Jardin F, et al. Tumor fragmentation estimated by volume surface ratio of tumors measured on 18F-FDG PET/CT is an independent prognostic factor of diffuse large B-cell lymphoma. Eur J Nucl Med Mol Imaging. 2018;45:1672-9.

24. Esfahani SA, Heidari P, Halpern EF, Hochberg EP, Palmer EL, Mahmood U. Baseline total lesion glycolysis measured with (18)F-FDG PET/CT as a predictor of progression-free survival in diffuse large B-cell lymphoma: a pilot study. Am J Nucl Med Mol Imaging. 2013;3:272-81.

25. Gallicchio R, Mansueto G, Simeon V, Nardelli A, Guariglia R, Capacchione D, et al. F-18 FDG PET/CT quantization parameters 
as predictors of outcome in patients with diffuse large B-cell lymphoma. Eur J Haematol. 2014;92:382-9.

26. Huang H, Xiao F, Han X, Zhong L, Zhong H, Xu L, et al. Correlation of pretreatm ent $18 \mathrm{~F}-\mathrm{FDG}$ uptake with clinicopathological factors and prognosis in patients with newly diagnosed diffuse large B-cell lymphoma. Nucl Med Commun. 2016;37:689-98.

27. Ilyas H, Mikhaeel NG, Dunn JT, Rahman F, Möller H, Smith D, et al. Is there an optimal method for measuring baseline metabolic tumor volume in diffuse large B cell lymphoma? Eur J Nucl Med Mol Imaging. 2019;46:520-1.

28. Jegadeesh N, Rajpara R, Esiashvili N, Shi Z, Liu Y, Okwan-Duodu $\mathrm{D}$, et al. Predictors of local recurrence after rituximab-based chemotherapy alone in stage III and IV diffuse large b-cell lymphoma: guiding decisions for consolidative radiation. Int J Radiat Oncol Biol Phys. 2015;92:107-12.

29. Kanoun S, Tal I, Berriolo-Riedinger A, Rossi C, Riedinger JM, Vrigneaud JM, et al. Influence of software tool and methodological aspects of total metabolic tumor volume calculation on baseline [18F] FDG PET to predict survival in Hodgkin lymphoma. PLoS One. 2015;10:1-15.

30. Kim CY, Hong CM, Kim DH, Son SH, Jeong SY, Lee SW, et al. Prognostic value of whole-body metabolic tumour volume and total lesion glycolysis measured on 18F-FDG PET/CT in patients with extranodal NK/T-cell lymphoma. Eur J Nucl Med Mol Imaging. 2013;40:1321-9.

31. Kim TM, Paeng JC, Chun IK, Keam B, Jeon YK, Lee SH, et al. Total lesion glycolysis in positron emission tomography is a better predictor of outcome than the International Prognostic Index for patients with diffuse large B cell lymphoma. Cancer. 2013;119: 1195-202.

32. Kwon SH, Kang DR, Kim J, Yoon JK, Lee SJ, Jeong SH, et al. Prognostic value of negative interim 2-[ 18 F]-fluoro-2-deoxy-dglucose $\mathrm{PET} / \mathrm{CT}$ in diffuse large B-cell lymphoma. Clin Radiol. 2016;71:280-6.

33. Lanic H, Mareschal S, Mechken F, Picquenot JM, Cornic M, Maingonnat $\mathrm{C}$, et al. Interim positron emission tomography scan associated with international prognostic index and germinal center B cell-like signature as prognostic index in diffuse large B-cell lymphoma. Leuk Lymphoma. 2012;53:34-42.

34. Lue KH, Wu YF, Liu SH, Hsieh TC, Chuang KS, Lin HH, et al. Prognostic value of pretreatment radiomic features of 18F-FDG PET in patients with Hodgkin lymphoma. Clin Nucl Med. 2019;44:E559-65

35. Mettler J, Müller H, Voltin CA, Baues C, Klaeser B, Moccia A, et al. Metabolic tumor volume for response prediction in advancedstage hodgkin lymphoma. J Nucl Med. 2019;60:207-11.

36. Mikhaeel NG, Smith D, Dunn JT, Phillips M, Møller H, Fields PA, et al. Combination of baseline metabolic tumour volume and early response on PET/CT improves progression-free survival prediction in DLBCL. Eur J Nucl Med Mol Imaging. 2016;43:1209-19.

37. Milgrom SA, Elhalawani H, Lee J, Wang Q, Mohamed ASR, Dabaja BS, et al. A PET radiomics model to predict refractory mediastinal Hodgkin lymphoma. Sci Rep. 2019;9:1-8.

38. Miyazaki Y, Nawa Y, Miyagawa M, Kohashi S, Nakase K, Yasukawa M, et al. Maximum standard uptake value of $18 \mathrm{~F}$ fluorodeoxyglucose positron emission tomography is a prognostic factor for progression-free survival of newly diagnosed patients with diffuse large B cell lymphoma. Ann Hematol. 2013;92:239-44.

39. Park S, Moon SH, Park LC, Hwang DW, Ji JH, Maeng CH, et al. The impact of baseline and interim PET/CT parameters on clinical outcome in patients with diffuse large B cell lymphoma. Am J Hematol. 2012;87:937-40.
40. Sasanelli M, Meignan M, Haioun C, Berriolo-Riedinger A, Casasnovas RO, Biggi A, et al. Pretherapy metabolic tumour volume is an independent predictor of outcome in patients with diffuse large B-cell lymphoma. Eur J Nucl Med Mol Imaging. 2014;41:2017-22.

41. Senjo H, Hirata K, Izumiyama K, Minauchi K, Tsukamoto E, Itoh $\mathrm{K}$, et al. High metabolic heterogeneity on baseline 18FDG-PET/CT scan as a poor prognostic factor for newly diagnosed diffuse large B-cell lymphoma. Blood Adv. 2020;4:2286-96.

42. Song MK, Yang DH, Lee GW, Lim SN, Shin S, Pak KJ, et al. High total metabolic tumor volume in PET/CT predicts worse prognosis in diffuse large B cell lymphoma patients with bone marrow involvement in rituximab era. Leuk Res. 2016;42:1-6.

43. Song MK, Chung JS, Lee JJ, Jeong SY, Lee SM, Hong JS, et al. Metabolic tumor volume by positron emission tomography/ computed tomography as a clinical parameter to determine therapeutic modality for early stage Hodgkin's lymphoma. Cancer Sci. 2013;104:1656-61.

44. Song MK, Chung JS, Shin HJ, Lee SM, Lee SE, Lee HS, et al. Clinical significance of metabolic tumor volume by PET/CT in stages II and III of diffuse large B cell lymphoma without extranodal site involvement. Ann Hematol. 2012;91:697-703.

45. Toledano MN, Desbordes P, Banjar A, Gardin I, Vera P, Ruminy P, et al. Combination of baseline FDG PET/CT total metabolic tumour volume and gene expression profile have a robust predictive value in patients with diffuse large B-cell lymphoma. Eur J Nucl Med Mol Imaging. 2018;45:680-8.

46. Tseng D, Rachakonda LP, Su Z, Advani R, Horning S, Hoppe RT, et al. Interim-treatment quantitative PET parameters predict progression and death among patients with hodgkin's disease. Radiat Oncol. 2012;7:5.

47. Xie M, Zhai W, Cheng S, Zhang H, Xie Y, He W. Predictive value of F-18 FDG PET/CT quantization parameters for progression-free survival in patients with diffuse large B-cell lymphoma. Hematology. 2016;21:99-105.

48. Zhang YY, Song L, Zhao MX, Hu K. A better prediction of progression-free survival in diffuse large B-cell lymphoma by a prognostic model consisting of baseline TLG and \% $\Delta$ SUVmax. Cancer Med. 2019;8:5137-47.

49. Zhou M, Chen Y, Huang H, Zhou X, Liu J, Huang G. Prognostic value of total lesion glycolysis of baseline F-fluorodeoxyglucose positron emission tomography/ computed tomography in diffuse large B-cell lymphoma other factors including MTV, National Comprehensive Cancer Network International Prognostic Ind. Oncotarget. 2016;7:83544-53.

50. Fletcher JW, Kinahan PE. PET/CT standardized uptake values (SUVs) in clinical practice and assessing response to therapy. Semin Ultrasound CT MR. 2010;31:496-505.

51. Adams MC, Turkington TG, Wilson JM, Wong TZ. A systematic review of the factors affecting accuracy of SUV measurements. Am J Roentgenol. 2010;195:310-20.

52. Fletcher JW, Kinahan PE. PET/CT standardized uptake values (SUVs) in clinical practice and assessing response to therapy. Semin Ultrasound CT MR. 2010;31:496-505.

53. Lambin P, Leijenaar RTH, Deist TM, Peerlings J, De Jong EEC, Van Timmeren J, et al. Radiomics: the bridge between medical imaging and personalized medicine. Nat Rev Clin Oncol. 2017;14:749-62.

54. Lundström CF, Gilmore HL, Ros PR. Integrated diagnostics: the computational revolution catalyzing cross-disciplinary practices in radiology, pathology, and genomics. Radiology. 2017;285:12-5. 
55. Barrington SF, Meignan M. Time to prepare for risk adaptation in lymphoma by standardizing measurement of metabolic tumor burden. J Nucl Med. 2019;60:1096-102.

56. Meignan M, Sasanelli M, Casasnovas RO, Luminari S, Fioroni F, Coriani $\mathrm{C}$, et al. Metabolic tumour volumes measured at staging in lymphoma: methodological evaluation on phantom experiments and patients. Eur J Nucl Med Mol Imaging. 2014;41:1113-22.

57. Burggraaff CN, Rahman F, Kaßner I, Pieplenbosch S, Barrington $\mathrm{SF}$, Jauw YWS, et al. Optimizing workflows for fast and reliable metabolic tumor volume measurements in diffuse large B cell lymphoma. Mol Imaging Biol. 2020;22:1102-10.

58. Vercellino L, Cottereau A-S, Casasnovas O, Tilly H, Feugier P, Chartier L, et al. High total metabolic tumor volume at baseline predicts survival independent of response to therapy. Blood. 2020;135:1396-405.

Publisher's note Springer Nature remains neutral with regard to jurisdictional claims in published maps and institutional affiliations. 